January 24,1995

\title{
Gyrokinetic Particle Simulation of Neoclassical Transport
}

\author{
Z. Lin, W. M. Tang, and W. W. Lee \\ Princeton Plasma Physics Laboratory \\ Princeton University \\ P.O. Box 451 \\ Princeton, NJ 08543-0451
}

\begin{abstract}
A time varying weighting $(\delta f)$ scheme for gyrokinetic particle simulation is applied to a steady state, multi-species simulation of neoclassical transport. Accurate collision operators conserving momentum and energy are developed and implemented. Simulation results using these operators are found to agree very well with neoclassical theory. For example, it is dynamically demonstrated in these multispecies simulations that like-particle collisions produce no particle flux and that the neoclassical fluxes are ambipolar for an ion-electron plasma. An important physics feature of the present scheme is the introduction of toroidal sheared flow to the simulations. Simulation results are in agreement with the existing analytical neoclassical theory of Hinton and Wong. The poloidal electric field associated with toroidal mass flow is found to enhance density gradient driven electron particle flux and the bootstrap current while reducing temperature gradient driven flux and current. Finally, neoclassical theory in steep gradient profile relevant to the edge regime is examined by taking into account finite banana width effects. In general, the present work demonstrates a valuable new capability for studying important aspects of neoclassical transport inaccessible by conventional analytical calculation processes.
\end{abstract}

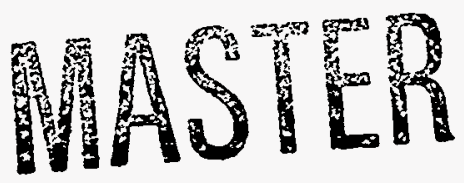




\section{INTRODUCTION}

It is generally acknowledged that neoclassical theory provides a useful lower bound for comparing confinement properties in magnetically confined plasmas. ${ }^{1}$ Standard neoclassical theory begins with the assumption of a static magnetic field equilibrium with no fluctuating fields. Recently several authors ${ }^{2-4}$ have pointed out that external electrostatic and magnetic fluctuations can strongly influence neoclassical transport. Another issue of current interest is the realistic extrapolation of the neoclassical bootstrap current into advanced tokamak operating regimes and assessing its efficacy for driving a steady state device. ${ }^{5,6}$ Neoclassical theory is also an important area of stellarator research since present-day experiments find that this type of transport is apparently dominant in the long mean free path regime. ${ }^{7}$ Analytical neoclassical theory has its limitation in all of the just noted situations due, for example, to complications introduced by realistic geometry. It is, therefore, of interest to systematically analyze this problem using particle simulation techniques. Potentially significant modifications associated with finite gyro-radius dynamics, energetic particle effects, sheared flows, and the influence of fluctuating electric fields can be examined with this approach. The scaling of the bootstrap current under realistic conditions in a steady-state tokamak can also be properly investigated. Finally, the fully 3dimensional non-axisymmetric nature of stellarator configurations can best be addressed by particle simulations.

The numerical simulation of neoclassical transport based on the driftkinetic formalism was carried out in early works by Tsang, et al. ${ }^{8}$ More recently, Wu and White ${ }^{9}$ used a Hamiltonian guiding center Monte-Carlo code to study the bootstrap current. $\mathrm{Ma}$, et al. ${ }^{10}$ developed a particle simulation scheme using the conventional gyrokinetic algorithm (total- $f$ ) and binary collisions. Our present work is intended to develop a tool which can be used for more comprehensive investigations. To this end, we have extended the gyrokinetic simulation techniques developed by Lee and cowork$\operatorname{ers}^{11-13}$ to a new regime of applicability. Specifically, the new $\delta f$ scheme is a fully dynamical, self-consistent and systematic approach, which has distinct numerical advantages over conventional simulation methods.

The approach in this paper differs from previous studies in a number of significant ways. First, the $\delta f$ scheme is a steady state simulation without profile relaxation effects, while a Monte-Carlo simulation will cause profile modification due to transport. Secondly, the noise level is greatly reduced in 


\section{DISCLAIMER}

This report was prepared as an account of work sponsored by an agency of the United States Government. Neither the United States Government nor any agency thereof, nor any of their employees, make any warranty, express or implied, or assumes any legal liability or responsibility for the accuracy, completeness, or usefulness of any information, apparatus, product, or process disclosed, or represents that its use would not infringe privately owned rights. Reference herein to any specific commercial product, process, or service by trade name, trademark, manufacturer, or otherwise does not necessarily constitute or imply its endorsement, recommendation, or favoring by the United States Government or any agency thereof. The views and opinions of authors expressed herein do not necessarily state or reflect those of the United States Government or any agency thereof. 


\section{DISCLAIMER}

\section{Portions of this document may be illegible in electronic image products. Images are produced from the best available original document.}


the present scheme compared to conventional particle simulations. Finally, momentum-conserving collision operators can be readily implemented using $\delta f$ scheme. The present work is the first steady state, multi-species simulation with ion dynamics retained and complete collision operators properly implemented. Simultaneously accounting for the ion and electron dynamics is very important because of the sensitivity of the ion response to effects such as sheared flows and finite gyroradius physics. This in turn can significantly modifies the electron transport through the collisional coupling and quasi-neutrality constrain.

In the usual $\delta f$ scheme for turbulence simulations, the distribution function is separated into a "known" or background $f_{0}$ and a perturbed part $\delta f$. When $\delta f \ll f_{0}$, the noise level is reduced by a factor of $\left(\delta f / f_{0}\right)^{2}$ compared to the total $f$ scheme. ${ }^{14,15}$ In the simulation of microturbulence, $f_{0}$ represents the background equilibrium distribution function and $\delta f$ accounts for the perturbation. In neoclassical transport, there are no fluctuations. Nevertheless, to facilitate the computations, the distribution function can still be separated into a Maxwellian $f_{0}$ plus a perturbed part, $\delta f$, with the perturbed part resulting from magnetic drifts and spatial inhomogeneity. We can then load a Maxwellian $f_{0}$ and calculate $\delta f$ as a time dependent quantity in the simulation. In this way, we can extend the $\delta f$ scheme to steady state simulation and study steady state phenomena by the initial value approach.

In the present paper, we extend the $\delta f$ schemes based on the small gyroradius ordering of drift kinetic equation to simulate steady state physics. The numerical scheme is benchmarked by using a simple model collision operator to study neoclassical transport. Simulations results of particle fluxes, energy fluxes, and bootstrap current are found to agree very well with standard neoclassical theory. Based on the approach adopted by Xu and Rosenbluth and later by Dimits and Cohen, ${ }^{16}$ accurate collision operators are developed and implemented. Specifically, all collisions conserve local momentum and energy, and the like-species collision operator properly annihilates the linearized shifted Maxwellian distribution. The relevance of momentum and energy conservation and the role of like-species collisions in neoclassical transport is explored in detail. Ion dynamics are self-consistently retained for the first time in these multi-species simulations, and it is dynamically demonstrated that (i) like-species collisions produce no particle flux; and (ii) neoclassical fluxes are automatically ambipolar for simple ion-electron plasma. Pure toroidal flows have also been introduced for the first time 
into these simulations. The trends predicted by the analytic neoclassical theory ${ }^{17}$ of large toroidal mass flow is confirmed. In the banana regime, the neoclassical enhancement of the viscosity is a Pfirsch-Schluter factor times the classical viscosity, and the enhancement of ion heat flux is observed. The direct effect of toroidal flow on electron particle transport is negligible when the Mach number is smaller than unity. Furthermore, the poloidal electric field associated with the flow is found to enhance the density-gradient-driven electron particle flux and bootstrap current, but reduce temperature gradient driven electron particle flux and the associated bootstrap current contribution. The effect on electron thermal fluxes is largely negligible. Finally, the neoclassical theory is re-examined in the steep gradient profile regime where the ion poloidal gyroradius is comparable to the equilibrium profile scale length. This finite banana width effect is studies both analytically and numerically. It is found that both the ion thermal flux and the toroidal mass flow are increased by factors of order $\left(\rho_{p} \kappa\right)^{2}$ when the finite banana width effects are taken into account, where $\rho_{p}$ is the ion poloidal gyroradius and $\kappa$ is the profile gradient. Future work using this new simulation technique to study various aspects of neoclassical transport will be discussed.

The rest of the paper is organized as following. Section II presents the basic formalism. Section III shows the results of benchmarking single species simulations. Accurate collision operators are developed in Section IV. The relevance of conservation properties of the collision operators is demonstrated in Section V. The effects of toroidal flow and the associated poloidal electric field are investigated in Section VI. Finally, neoclassical theory for the steep equilibrium gradient profile case is examined in Section VII with finite banana width effect retained. Section VIII summarizes the main findings of the present work and comments on future studies.

\section{II. $\delta f$ SCHEME FOR NEOCLASSICAL TRANSPORT}

\section{A. Basic Formalism}

To illustrate the basic principle behind the computational approach developed to address the neoclassical transport problem, we begin by considering a simple steady state plasma with static magnetic field $B \hat{b}$ and no electric field. The usual drift kinetic equation for a guiding center distribution function $f(E, \mu, \mathbf{x})$, where $E$ is particle kinetic snergy, $\mu$ is magnetic moment and $\mathrm{x}$ is guiding center coordinate, has the form, ${ }^{1}$ 


$$
\frac{\partial f}{\partial t}+\left(v_{\|} \hat{\mathbf{b}}+\mathbf{v}_{d}\right) \cdot \frac{\partial f}{\partial \mathbf{x}}-C(f)=0
$$

where $\mathrm{v}_{d}$ is guiding center drift velocity, $C$ is the drift kinetic collision operator. In the steady state,

$$
\frac{\partial f}{\partial t}=0
$$

The basic small expansion parameter is

$$
\delta=\frac{\rho_{p}}{R_{0}}
$$

where $\rho_{p}$ is the ion poloidal gyroradius and $R_{0}$ is the major radius of the torus.

Since the drift term $\mathbf{v}_{d}$ is smaller than transit term $v_{\|}$by a factor of $\delta,{ }^{1}$ a perturbation expansion base on $\delta$ ordering is appropriate; i.e. ,

$$
f=f_{0}+f_{1}+\cdots \text {. }
$$

The zeroth order equation then becomes

$$
v_{\|} \hat{\mathbf{b}} \cdot \frac{\partial f_{0}}{\partial \mathrm{x}}-C\left(f_{0}\right)=0
$$

with its solution being a local Maxwellian

$$
f_{0}=n_{0} F_{m}=\frac{n_{0}}{\pi^{\frac{3}{2}} v_{t h}^{3}} e^{-\frac{v^{2}}{v_{t h}^{2}}}
$$

The first order equation is

$$
v_{\|} \hat{\mathbf{b}} \cdot \frac{\partial f_{1}}{\partial \mathbf{x}}-C\left(f_{1}\right)=-\mathbf{v}_{d} \cdot \frac{\partial f_{0}}{\partial \mathbf{x}}
$$

Together with the solubility conditions from the second order equation, we can solve for $f_{1}$ in term of linear functions of $f_{0}$. The formal solution is

$$
f_{1}=\left(v_{\|} \hat{\mathbf{b}} \cdot \frac{\partial}{\partial \mathbf{x}}-C\right)^{-1} \mathbf{v}_{d} \cdot \vec{\kappa} f_{0}
$$

where

$$
\vec{\kappa}=\left[\kappa_{n}+\left(\frac{v^{2}}{v_{t h}^{2}}-\frac{3}{2}\right) \kappa_{t}\right] \vec{e}_{r}
$$


with $\kappa_{n}$ and $\kappa_{t}$ representing the inverse of density and temperature scale length, respectively.

In order to utilize particle simulation techniques to solve the first order equation, Eq. 3, we adopt the following approach. This equation is solved numerically by following the zeroth order guiding center trajectory in phase space, which is defined by the characteristics of zeroth order equation, Eq. 2. To cast the drift kinetic equation in a form suitable for particle pushing, we make a transformation to guiding center phase space variables $\left(\mu, v_{\|}, \mathbf{x}\right)$,

$$
f(E, \mu, \mathbf{x}) \rightarrow f\left[v_{\|}(E, \mu, \mathbf{x}), \mu, \mathbf{x}\right],
$$

which leads to,

$$
\left(\frac{\partial f}{\partial \mathbf{x}}\right)_{E, \mu}=\left(\frac{\partial f}{\partial \mathbf{x}}\right)_{\mu, v_{\|}}+\left(\frac{\partial f}{\partial v_{\|}}\right)_{\mu, \mathbf{x}}\left(\frac{\partial v_{\|}}{\partial \mathbf{x}}\right)_{E, \mu} .
$$

After defining

$$
a_{\|}=v_{\|} \hat{\mathrm{b}} \cdot\left(\frac{\partial v_{\|}}{\partial \mathrm{x}}\right)_{E, \mu},
$$

Eq. 2 and Eq. 3 can then be expressed as

$$
\left\{\begin{array}{l}
\frac{\partial f_{0}}{\partial t}+v_{\|} \hat{\mathrm{b}} \cdot \frac{\partial f_{0}}{\partial \mathbf{x}}+a_{\|} \frac{\partial f_{0}}{\partial v_{\|}}-C\left(f_{0}\right)=0 \\
\frac{\partial f_{1}}{\partial t}+v_{\|} \hat{\mathrm{b}} \cdot \frac{\partial f_{1}}{\partial \mathbf{x}}+a_{\|} \frac{\partial f_{1}}{\partial v_{\|}}-C\left(f_{1}\right)=\mathrm{v}_{d} \cdot \vec{\kappa} f_{0} .
\end{array}\right.
$$

With regard to the physical interpretation of these equations, we note that a steady state distribution function close to a local Maxwellian has been considered. It can then be linearized and separated into a zeroth order background Maxwellian and a first order perturbation part. The background inhomogeneity only comes in through the drift term in the steady state drift kinetic equation. Since this drift term is much smaller than the transit term, we can separate it out and treat it as a source term in the linearized drift kinetic equation.

Eqs. 4 illustrate the desired form needed for numerical simulation. It contains all the important neoclassical effects, i.e., the $\mathbf{v}_{d}$ term accounting for magnetic gradient and curvature drifts, and the $a_{\|}$term representing the mirror force term which gives rise to particle trapping. These equations can be readily solved utilizing the linearized weighting scheme of Dimits and Lee. ${ }^{12}$ In this linearized scheme, particles are pushed by following the 
zeroth order guiding center trajectory without the gradient and curvature drifts which are taken into account in the first order equation. $f_{1}$ (or $\delta f$ in the usual notation of gyrokinetic simulation) is solved by integration along the particle trajectory. In this way we can solve the steady state problem by initial value methods. The steady state solution of $f_{1}$ is obtained after several characteristic time periods governed by the left side of Eq. 4. This is the collisional time in the banana regime and the parallel diffusion time in the collisional regime.

Magnetic surface averaged neoclassical fluxes and diffusion coefficients only depend on local density, temperature and their gradients. Hence, instead of loading a real profile, we can load a normalized $f_{0}$ which is uniform in space with density and temperature equal to those of the magnetic surface on which we are computing the neoclassical fluxes.

\section{B. Finite Banana Width Effects}

In order to take into account finite banana width effects which usually are not included in the neoclassical theory, we need to follow a more exact guiding center motion. Thus, the drift term $\mathbf{v}_{d}$ must be retained to the leading order in drift kinetic equation,

$$
\frac{\partial f}{\partial t}+\left(v_{\|} \hat{\mathbf{b}}+\mathbf{v}_{d}\right) \cdot \frac{\partial f}{\partial \mathbf{x}}+a_{\|} \frac{\partial f}{\partial v_{\|}}-C(f)=0
$$

For

$$
f=f_{0}+\delta f
$$

$f_{0}$ now satisfies

$$
\frac{\partial f_{0}}{\partial t}+v_{\|} \hat{\mathbf{b}} \cdot \frac{\partial f_{0}}{\partial \mathbf{x}}+a_{\|} \frac{\partial f_{0}}{\partial v_{\|}}-C\left(f_{0}\right)=0
$$

The governing equation for the perturbation is then become,

$$
\frac{\partial \delta f}{\partial t}+\left(v_{\|} \hat{\mathbf{b}}+\mathbf{v}_{d}\right) \cdot \frac{\partial \delta f}{\partial \mathbf{x}}+a_{\|} \frac{\partial \delta f}{\partial v_{\|}}-C(\delta f)=\mathbf{v}_{\mathbf{d}} \cdot \kappa f_{0} .
$$

Equation 6 can be efficiently solved using the nonlinear weighting scheme of Parker and Lee ${ }^{13}$. This is accomplished by first defining a symbolic operator

$$
\frac{D}{D t} \equiv \frac{\partial}{\partial t}+\left(v_{\|} \hat{\mathbf{b}}+\mathbf{v}_{\mathbf{d}}\right) \cdot \frac{\partial}{\partial \mathbf{x}}+a_{\|} \frac{\partial}{\partial v_{\|}}-C,
$$


and weight $w$

$$
w \equiv \frac{\delta f}{f}
$$

Then, we have

$$
\frac{D w}{D t}=\mathbf{v}_{\mathbf{d}} \cdot \kappa \frac{f_{0}}{f}=(1-w) \mathbf{v}_{\mathbf{d}} \cdot \kappa .
$$

If a Maxwellian distribution is considered,

$$
f(t=0)=f_{0}=F_{m},
$$

for

$$
f=\sum_{i=1}^{N} \delta\left(\mathbf{Z}-\mathbf{Z}_{i}\right),
$$

the solution of $\delta f$ is then given by

$$
\delta f=\sum_{i=1}^{N} w_{i} \delta\left(\mathbf{Z}-\mathbf{Z}_{i}\right)
$$

where $\mathbf{Z}$ represents the five-dimension phase space variables $\left(\mu, v_{\|}, \mathbf{x}\right)$.

When ion-electron collisions are neglected, the solution of $f_{0}$ can be generalized to a shifted Maxwellian $F_{s m}\left(v_{\|}-v_{\| 0}\right)$ with mean velocity $v_{\| 0}$. The associated equilibrium gradient scale parameter $\kappa$ is defined as,

$$
\kappa=\kappa_{n}+\left(\frac{v^{2}}{v_{t h}^{2}}-\frac{3}{2}\right) \kappa_{t}+\frac{2\left(v_{\|}-v_{\| 0}\right) v_{\| 0}}{v_{t h}^{2}} \kappa_{v},
$$

where $\kappa_{v}$ is the inverse of flow velocity scale length.

\section{BENCHMARKING THE NUMERICAL SCHEME}

To benchmark the numerical scheme, a simulation in toroidal geometry is carried out using a model collision operator. Consider an axisymmetric toroidal geometry with circular cross section. The magnetic field can be written as

$$
\mathbf{B}=B_{T}(r) \hat{\phi}+B_{P}(r) \hat{\theta},
$$

where $r, \hat{\phi}$ and $\hat{\theta}$ are, respectively, the minor radius, the toroidal and the poloidal angles. Here $B_{T}=B_{0} / h, B_{P}=B_{p 0} / h$ with $h$ defined as

$$
h \equiv 1+\frac{r}{R_{0}} \cos \theta \equiv 1+\epsilon \cos \theta
$$


and $R_{0}$ is the major radius. $B_{0}$ and $B_{p 0}$ are related by the safety factor $q$,

$$
q=\frac{r B_{0}}{R_{0} B_{p 0}}
$$

For numerical simplicity, we use the familiar Lorentz model without velocity dependence, i.e.,

$$
C=\nu \hat{\mathrm{L}}=\nu \frac{1}{2} \frac{\partial}{\partial \xi}\left(1-\xi^{2}\right) \frac{\partial}{\partial \xi},
$$

where $\nu$ is the collision frequency, $\hat{\mathbf{L}}$ is the pitch angle scattering operator, and $\xi$ is the particle pitch with respect to magnetic field line,

$$
\xi=\frac{v_{\|}}{v} \text {. }
$$

\section{A. Analytical Neoclassical Theory}

In order to provide the benchmarks for the simulation results, we analytically calculate the neoclassical fluxes for the model collision operators in both collisionless and collisional limits. We begin with the first order steady state drift kinetic equation, Eq. 3, with the drift velocity ${ }^{1}$ and the collision operator written as

$$
v_{d r}=\frac{v_{\|}}{\Omega_{p}} \hat{\mathrm{b}} \cdot \nabla\left(h v_{\|}\right),
$$

and

$$
C=\nu 2 h \xi \frac{\partial}{\partial \lambda} \lambda \xi \frac{\partial}{\partial \lambda}
$$

respectively. Here $\Omega_{p}=e B_{p 0} / m c$ and $\lambda=h\left(1-\xi^{2}\right)$.

1. Banana Regime

In the banana ordering, $f_{1}$ can be expanded as;

$$
f_{1}=f_{1}^{(0)}+f_{1}^{(1)}+\cdots,
$$

where the smallness parameter is,

$$
\frac{f_{1}^{(1)}}{f_{1}^{(0)}} \sim \frac{\nu}{\omega_{t}},
$$


where $\omega_{t}$ is the transit frequency. The zeroth order equation becomes

$$
v_{\|} \hat{\mathbf{b}} \cdot \nabla\left(f_{1}^{(0)}-\frac{v \kappa}{\Omega_{p}} h \xi\right) F_{m}=0
$$

or

$$
f_{1}^{(0)}=\frac{v \kappa}{\Omega_{p}} F_{m}\left(h \xi+\hat{f}_{1}\right)
$$

where

$$
\frac{\partial \hat{f}_{1}}{\partial \theta}=0
$$

Application of the annihilator

$$
\oint \frac{d \theta}{v_{\|}}
$$

to the first order equation,

$$
v_{\|} \hat{\mathbf{b}} \cdot \nabla f_{1}^{(1)}-C\left(f_{1}^{(0)}\right)=0
$$

leads to the following solubility condition:

$$
\oint \frac{d \theta}{v_{\|}} C\left(f_{1}^{(0)}\right)=0
$$

Thus, we find for $\hat{f}_{1}$

$$
\frac{\partial \hat{f}_{1}}{\partial \lambda}=\frac{1}{2<\xi>} H\left(h_{\min }-\lambda\right)
$$

where $\mathrm{H}$ is a step function, and $h_{\min }=1-\epsilon$ defines the boundary between trapped and passing particles. With $f_{1}^{(0)}$ and first order equation for $f_{1}^{(1)}$ specified, we can calculate the corresponding neoclassical fluxes:

$$
\left\{\begin{array}{l}
\Gamma=<\int d^{3} v v_{d r} f_{1}>=\frac{3}{8} I_{1} \nu \rho^{2} \frac{q^{2}}{\epsilon^{2}} n\left(\kappa_{n}+\kappa_{T}\right), \\
Q=<\int d^{3} v \frac{1}{2} m v^{2} v_{d r} f_{1}>=\frac{5 T}{2}\left(\Gamma+\frac{3}{8} I_{1} \nu \rho^{2} \frac{q^{2}}{\epsilon^{2}} n \kappa_{T}\right), \\
j_{b}=<\int d^{3} v \frac{v_{\|}}{h} f_{1}>=f_{t} \frac{c}{B_{p 0}} \frac{d p}{d r}=\frac{3}{4} I_{3} \frac{c}{B_{p 0}} \frac{d p}{d r},
\end{array}\right.
$$

where $\langle\cdots\rangle$ represents the flux surface averaging, $\int_{0}^{2 \pi} h d \theta / 2 \pi$. Here, $\Gamma$ is the particle flux, $Q$ is the energy flux and $j_{b}$ is the bootstrap 
current with $\rho$ being the thermal gyroradius $\left(\rho=m v_{t h} c / e B_{0}\right), f_{t}$ is the fraction of trapped particles, and to the lowest order in $\epsilon$,

$$
I_{1}=I_{3}=1.38 \sqrt{2 \epsilon}
$$

For a finite $\epsilon$, as pointed out by $\mathrm{Wu}$ and White, ${ }^{9}$ the next order correction to the trapped particle fraction can be estimated heuristically by requiring $f_{t}(\epsilon=1)=1$. This yields:

$$
f_{t}=1.46 \sqrt{\epsilon}-0.46 \epsilon \text {. }
$$

We note that to measure local (not volume averaged) current density, it is essential to calculate $\left\langle\int d^{3} v f_{1} v_{\|} / h>\right.$ rather than $\left\langle\int d^{3} v f_{1} v_{\| l}\right\rangle$.

2. Collisional Regime

In collisional regime, $f_{1}$ can be expanded as:

$$
f_{1}=f_{1}^{(-1)}+f_{1}^{(0)}+f_{1}^{(1)}+\cdots,
$$

where the smallness parameter is

$$
\frac{f_{1}^{(0)}}{f_{1}^{(-1)}} \sim \frac{\omega_{t}}{\nu}
$$

The associated lowest order equation is

$$
C\left(f_{1}^{(-1)}\right)=0
$$

or

$$
f_{1}^{(-1)}=f_{1}^{(-1)}(E, r, \theta) .
$$

The zeroth order equation is then given by

$$
C\left(f_{1}^{(0)}\right)=v_{\|} \hat{\mathrm{b}} \cdot \nabla f_{1}^{(-1)}
$$

This yields

$$
f_{1}^{(0)}=-\frac{v \xi}{\nu} \hat{\mathrm{b}} \cdot \nabla f_{1}^{(-1)}
$$

For the first order equation,

$$
C\left(f_{1}^{(1)}\right)=v_{\|} \hat{\mathbf{b}} \cdot \nabla f_{1}^{(0)}-\frac{v \kappa}{\Omega_{p}} F_{m} v_{\|} \hat{\mathbf{b}} \cdot \nabla(h \xi),
$$


$f_{1}^{(0)}$ can then be substituted and the annihilator

$$
\int_{0}^{h} d \lambda
$$

can be applied together with

$$
\int_{0}^{2 \pi} \frac{d \theta}{h}
$$

to give

$$
\hat{\mathbf{b}} \cdot \nabla f^{(-1)}=\frac{\nu \kappa}{\Omega_{p}} F_{m}\left(\frac{<h^{-2}>^{-1}}{h}-h\right) .
$$

The corresponding neoclassical fluxes are

$$
\left\{\begin{array}{l}
\Gamma=\nu q^{2} \rho^{2} n\left(\kappa_{n}+\kappa_{T}\right), \\
Q=\frac{5 T}{2}\left(\Gamma+\nu q^{2} \rho^{2} n \kappa_{T}\right) .
\end{array}\right.
$$

\section{B. Simulation Results}

In this benchmark simulation, we consider a static magnetic field and no electric field. A uniform Maxwellian of electrons is loaded over an annulus section of torus. We follow the electron guiding center trajectories and treat the ions as a cold Maxwellian background. The simplified Lorentz collision operator is implemented by the utilizing the Monte-Carlo pitch angle scattering model,

$$
\left.\xi=\xi_{0}(1-\nu \Delta t)+(R-0.5)\left[12\left(1-\xi_{0}^{2}\right) \nu \Delta t\right)\right]^{\frac{1}{2}},
$$

where $\xi$ and $\xi_{0}$ are pitch angles after and before collisions, respectively, $\Delta t$ is the time step, and $R$ is a uniform random number between 0 and 1 .

The neoclassical fluxes are measured within an annulus centered by a magnetic surface. Results from the 3-D toroidal code are shown in Figs. 1, 2 , and 3 , where we show the collision fiequency dependence of, respectively, particle flux $\Gamma$, energy flux $Q$ and bootstrap current $j_{b}$ ( $j_{b}$ is normalized by the collisionless limit value $j_{0}$ ). Results from the analytical neoclassical calculations using the same model collision operator are also plotted on these 
figures for comparison purpose. The effective collision frequency is defined by

$$
\nu^{*}=\frac{\nu \sqrt{2} q R_{0}}{\epsilon^{\frac{3}{2}} v_{t h}} .
$$

Throughout this paper, we use the gyrokinetic normalization of $B_{0}=T_{e}=$ $m_{i}=1$. Key parameters in the simulations are: $R_{0}=512, \epsilon=0.213$, $q=2.5, \kappa_{n}=0.02$, and $\kappa_{t}=0$.

The simulation results agree very well with analytic theory in both the collisional and the collisionless limits where analytical results are valid. This numerical scheme is sufficiently accurate to actually measure $\delta f$. To demonstrate that the present computational scheme correctly represents the physics, we now examine the neoclassical transport in some details in these two limits of collisionality.

1. Collisional Limit

In the collisional regime, $f_{1}$ can be written as

$$
f_{1}=\delta n(r, \theta) F_{m}+\frac{2 u(r, \theta) v_{\|}}{v_{t h}^{2}} F_{m}
$$

where $\delta n$ is local density perturbation, and $u$ is the parallel flow velocity. By expanding $\delta n$ and $u$ in poloidal harmonics,

$$
\begin{aligned}
\delta n & =\sum_{m=0}^{\infty} \delta n_{m}(r) e^{i m \theta}, \\
u & =\sum_{m=0}^{\infty} u_{m}(r) e^{i m \theta},
\end{aligned}
$$

and accurate to the lowest order in $\epsilon$ for Eq. 9, the solutions are

$$
\begin{aligned}
f_{1}^{(-1)} & =\cos \theta \frac{2 q^{2} R_{0} \nu \kappa}{\Omega} F_{m} \\
f_{1}^{(0)} & =\cos \theta \frac{2 q v_{\|} \kappa}{\Omega} F_{m}
\end{aligned}
$$

where $\Omega \equiv e B_{0} / m c$. Thus,

$$
\delta n_{1}=\frac{2 q^{2} R_{0} \nu \kappa}{\Omega}
$$


and

$$
u_{1}=\frac{q \kappa}{\Omega} .
$$

Good agreement is obtained for the amplitudes of $\delta n_{1}$. and $u_{1}$ between analytic theory and simulation results as depicted in in Fig. 4.

Diamagnetic current driven by background pressure gradient produces a parallel flow (Pfirsch-Schlüter current or return current) in the collisional limit as required by the quasineutrality condition. Due to the short mean mean path of the particles, this return current produces a first order pressure variation within the magnetic surface. The resulting diamagnetic-type drift gives rise to a neoclassical fluxes. Here the local Maxwellian $f_{1}^{(-1)}$ represents the pressure variation, and the shifted Maxwellian $f_{1}^{(0)}$ accounts for the parallel return current. In the simulation, $f_{1}$ reaches a steady state solution when the friction force on the return current due to collisions is balanced by the driving force from the gradient of the pressure perturbation. A plot of time history of the particle flux is shown in Fig. 5. It is found that the particles reach steady state in a few parallel diffusion times.

\section{Collisionless Limit}

In the collisionless (banana) regime, the pitch angle scattering pumps trapped particle into the untrapped population. At a giving magnetic surface, trapped particles with opposite parallel velocities comes from opposite sides of the surface, respectively, and hence carry different parallel momentum due to the density gradient. The circulating particles gain this momentum due to the detrapping process, and the resulting parallel flow give rise to a bootstrap current. On the other hand, the circulating particles lose momentum due to ion-electron collisions. When the friction force of the collisions balances the driving force of the density gradient, $f_{1}$ reaches a steady state solution. In this collisionless limit particles move freely along the magnetic field line to maintain uniform pressure, but the drift motions perturb free particle motion and give rise to stress anisotropy. The neoclassical fluxes again result from the diamagnetic outward drift due to this stress anisotropy. A example of the time history of the bootstrap current is shown in Fig. 6 where it is illustrated that this current reaches steady state in a few collision times. 


\section{Lorentz Model}

Since the Lorentz model is extensively used in neoclassical theory, it is interesting to assess its accuracy for computing the actual transport. In Fig. 7 the particle flux (represented by $X$ ) obtained by using Lorentz model is compared to theoretical fluxes calculated with the full Fokker-Planck operator. We note that in the collisional limit, the Lorentz model is quite adequate to produce the neoclassical fluxes. However it generally gives smaller fluxes in the banana regime where electron-electron collision cannot be ignored.

To include electron-electron collisions in the banana regime, note that only the pitch angle scattering part is important. It is convenient to adopt the model pitch angle scattering operator from Hinton and Hazeltine's review paper;

$$
C=\left(\nu_{e e}+\nu_{e i}\right) \hat{L}
$$

where,

$$
\begin{aligned}
\nu_{e i} & =\frac{3 \pi}{4 \tau_{b}}\left(\frac{v_{t h}}{v}\right)^{3}, \\
\nu_{e e} & =\frac{3 \pi}{4 \tau_{b}}\left(\frac{v_{t h}}{v}\right)^{3} \phi\left(\frac{v}{v_{t h}}\right), \\
\phi(x) & =\left(1-\frac{1}{2 x^{2}}\right) \operatorname{erf}(x)+\frac{e^{-x^{2}}}{\pi^{1 / 2} x}
\end{aligned}
$$

and $\operatorname{erf}(x)$ is the error function. The results of particle flux are also shown in Fig. 7 (represented by + ). This form is accurate in the small collision frequency limit but overestimates the fluxes in the more collisional regimes.

\section{FOKKER-PLANCK COLLISION OPERATION}

Realistic gyrokinetic particle simulation requires the implementation of accurate collision operators with all conservation properties retained. As shown by analytical theory, momentum conservation can play an important role in neoclassical transport. Therefore, this problem provides a relatively simple yet direct physical test for collision operators conserving momentum and energy. Appropriate accurate collisions operators conserving momentum and energy for a simple (electron-ion plasma) plasma are developed in this section. 


\section{A. Like-Species Collision Operators}

Following the approach introduced by $\mathrm{Xu}$ and Rosenbluth ${ }^{18}$ and then modified by Dimits and Cohen, ${ }^{16}$ we have developed a like-species particle collision operator which can (i) conserve all the collisional invariants(particle number, momentum and energy); and (ii) annihilate a shifted Maxwellian equilibrium distribution with small mean velocity. In dealing with collisions of test particles $(\alpha)$ with background particles $(\beta)$, we begin with the Rosenbluth potential, assume both distribution functions $f^{\alpha}$ and $f^{\beta}$ to be close to Maxwellian, linearize, and keep terms responsible for momentum and energy conservation. The linearized operator is ${ }^{19,20}$

$C\left(\delta f^{\alpha}\right)=P\left(F_{m}, \delta f^{\beta}\right)+\frac{\partial}{\partial \mathbf{v}} \cdot\left(\mathbf{v} F \delta f^{\alpha}\right)+\frac{1}{2} \frac{\partial^{2}}{\partial \mathbf{v} \partial \mathbf{v}}:\left[G\left(\mathbf{I} v^{2}-\mathbf{v v}\right)+H \mathbf{v v}\right] \delta f^{\alpha}$

where the first term $P$ accounts for the momentum and energy conservation, and the other two terms are the test particle drag and diffusion parts. Functions $F, G, H$ are defined by

$$
\begin{aligned}
F & =\left(1+\frac{m_{\alpha}}{m_{\beta}}\right) \phi(x) \nu_{0}, \\
G & =\left[\left(1-\frac{1}{2 x}\right) \phi(x)+\frac{d \phi(x)}{d x}\right] \nu_{0}, \\
H & =\frac{1}{x} \phi(x) \nu_{0},
\end{aligned}
$$

respectively. Here $x=v^{2} / v_{t h \beta}^{2}$ and $\phi(x)$ is the Maxwellian integral defined by

$$
\phi(x)=\frac{2}{\sqrt{\pi}} \int_{0}^{x} e^{-t} \sqrt{t} d t .
$$

The basic collision frequency here is defined by

$$
\nu_{0}=\frac{4 \pi n_{\beta} q_{\alpha}^{2} q_{\beta}^{2} \ln \Lambda_{\alpha \beta}}{m_{\alpha}^{2} v^{3}} .
$$

The diffusion tensor can be diagonalized by transforming to the coordinate system $\mathbf{w}$ in which the $z$-axis is the direction of the test particle velocity, $\mathbf{w}_{\|}=\mathbf{w}_{z}=\mathbf{v}$. This lead to

$C\left(\delta f^{\alpha}\right)=P\left(F_{m}, \delta f^{\beta}\right)+\frac{\partial}{\partial w_{\|}}\left(v F \delta f^{\alpha}\right)+\frac{1}{2} \frac{\partial^{2}}{\partial w_{\|}^{2}}\left(v^{2} H \delta f^{\alpha}\right)+\frac{1}{2}\left(\frac{\partial^{2}}{\partial w_{x}^{2}}+\frac{\partial^{2}}{\partial w_{y}^{2}}\right)\left(v^{2} G \delta f^{\alpha}\right)$ 
where $w_{x}$ and $w_{y}$ are orthogonal to $w_{\|}$and to each other. The test particle part can be readily implemented in this diagonalized form. ${ }^{21}$

In the drift kinetic limit, we can transform the velocity space coordinate to cylindrical coordinate $\left(v_{\|}, v_{\perp}, \phi\right)$ with $\phi$ representing gyro-angle. After averaging over gyrophase $\phi$, we have (now $\delta f$ is guiding center distribution function),

$$
\begin{aligned}
C(\delta f)= & \frac{\partial}{\partial v_{\|}}\left(\nu_{s \|} \delta f\right)+\frac{\partial}{\partial v_{\perp}{ }^{2}}\left(\nu_{s \perp} \delta f\right)+\frac{\partial^{2}}{\partial v_{\|} \partial v_{\perp}^{2}}\left(\nu_{\| \perp} \delta f\right) \\
& +\frac{1}{2} \frac{\partial^{2}}{\partial v_{\|}^{2}}\left(\nu_{\|} \delta f\right)+\frac{1}{2} \frac{\partial^{2}}{\left(\partial v_{\perp}^{2}\right)^{2}}\left(\nu_{\perp} \delta f\right)+P
\end{aligned}
$$

where the collision coefficients are

$$
\begin{aligned}
\nu_{s \|} & =v_{\|} F, \\
\nu_{s \perp} & =2 v_{\perp}^{2} F-v_{\perp}^{2} H-\left(2 v_{\|}^{2}+v_{\perp}^{2}\right) G, \\
\nu_{\|} & =v_{\|}^{2} H+v_{\perp}^{2} G, \\
\nu_{\perp} & =4 v_{\perp}^{2}\left(v_{\perp}^{2} H+v_{\|}^{2} G\right), \\
\nu_{\| \perp} & =2 v_{\perp}^{2} v_{\|}(H-G) .
\end{aligned}
$$

The test particle drag and diffusion terms can be implemented by utilizing the following Monte-Carlo method ${ }^{21,18,16}$;

$$
\begin{aligned}
v_{\|}= & v_{\| 0}-\nu_{s \|} \Delta t+\sqrt{12}\left(R_{1}-0.5\right) \sqrt{\nu_{\|} \Delta t} \\
v_{\perp}{ }^{2}= & v_{\perp 0}^{2}-\nu_{s \perp} \Delta t+\sqrt{12}\left(R_{2}-0.5\right) \sqrt{\left(\nu_{\perp}-\frac{\nu_{\| \perp}^{2}}{\nu_{\|}}\right) \Delta t} \\
& +\sqrt{12}\left(R_{1}-0.5\right) \frac{\nu_{\| \perp}}{\nu_{\|}} \sqrt{\nu_{\|} \Delta t},
\end{aligned}
$$

where $R_{1}, R_{2}$ are two independent uniform random numbers.

In particle simulations using the $\delta f$ scheme, the momentum and energy conservation term $P$ can be readily implemented. This term has been calculated analytically by $\mathrm{Xu}$ and Rosenbluth. ${ }^{18}$ Since it appears as a source term in the linearized gyrokinetic equation, Dimits and Cohen ${ }^{16}$ implement this term by changing particle weights to ensure the conservation of first order momentum and energy,

$$
\Delta w=-\mathbf{v} \cdot \delta \mathbf{P}-\left(\frac{v^{2}}{v_{t h \alpha}^{2}}-\frac{3}{2}\right) \delta E
$$


where $\delta P$ and $\delta E$ are weighted changes of the momentum $\left(\Delta \mathbf{v}_{i}\right)$ and energy $\left(\Delta v_{i}^{2}\right)$, respectively, of test particles due to test-particle collisions; i.e. ,

$$
\delta \mathbf{P}=\frac{2}{n v_{t h \alpha}^{2}} \sum_{i} w_{i} \Delta \mathbf{v}_{\mathbf{i}}
$$

and

$$
\delta E=\frac{2}{3 n v_{t h \alpha}^{2}} \sum_{i} w_{i} \Delta v_{i}^{2} .
$$

For like-species collisions, the collisional steady state solution is a shifted Maxwellian $F_{s m}=F_{m}\left(v-v_{\| 0}\right)$, and the collision operators should accordingly annihilate this function. However, the implementation of Eq. 15 fails to maintain a shifted Maxwellian. This method compensates the test-particle momentum and energy loss due to test-particle collisions by putting them back into particle weights. However, this approach does not take into account the velocity dependence of the momentum and energy loss rates generated by collisions. As a result, the shifted Maxwellian is distorted in velocity space, and only three velocity moments $\left(\left\langle v^{0}\right\rangle,\langle\mathrm{v}\rangle,\left\langle v^{2}\right\rangle\right)$ are conserved. As demonstrated in the next section, application of this procedure to the neoclassical transport problem will give incorrect energy flux, which is a quantity associated with third and higher order velocity moments.

Note that for small mean velocity $\left(v_{\| 0} \ll v_{t h}\right)$, a linearized shifted Maxwellian is linear both in $\mathbf{v}$ and $v^{2}$ to second order in $v_{\| 0} / v_{t h}$. We can therefore maintain a linearized shifted Maxwellian by restoring the momentum and energy according to their loss rates. The loss rates of momentum and energy ${ }^{19}$ can be readily calculated from Eq. 12:

$$
\begin{aligned}
\frac{d v}{d t} & =-F \mathbf{v}, \\
\frac{d v^{2}}{d t} & =-(2 F-2 G-H) v^{2} .
\end{aligned}
$$

Substituting the functions $F, H$ and $G$ defined in Eq. 11, we now can implement conservation properties with the correct velocity dependence,

$$
\Delta w=-3 \sqrt{\frac{\pi}{2}} \phi(x)\left(\frac{v_{t h \alpha}}{v}\right)^{3} \mathbf{v} \cdot \delta \mathbf{P}-3 \sqrt{\frac{\pi}{2}}\left[\phi(x)-\frac{d \phi(x)}{d x}\right] \frac{v_{t h \alpha}}{v} \delta E
$$

with $\delta \mathbf{P}$ and $\delta E$ determined by Eq. 17 .

Eqs. 13 and 18 represent an appropriate set of Fokker-Planck collision operators. It is obvious that these operators conserve the collisional invariants and properly annihilate a Maxwellian. To test the second property, a 
shifted Maxwellian $\delta f^{\alpha}=2 v_{\|} v_{0} / v_{t h \alpha}^{2}$ is loaded and subjected to the test particles drag and diffusion processes (Eq. 13). We then comparing the results obtained from applying Eq's (15) and (18), respectively. The shifted Maxwellian $\delta f^{\alpha}$ after three collisional times is shown in Fig. 8. It is clearly evident here that results using our new formulation, i.e., Eq. 18, (represented by solid line) maintains the form of the shifted Maxwellian $\delta f^{\alpha}$, while result from the application of Eq. 15 (represented by dotted line) fails to do so. The older model pumps the momentum from low velocity particles to high velocity particles and generates an increase in the higher velocity moments associated with the momentum accumulation of these high velocity particles. Fig. 9 compares the third velocity moment history of the same shifted Maxwellian under these two schemes.

\section{B. Inter-Species Collision Operators}

Collisions between ions and electrons can be simplified by neglecting the mass of the electron. In the ion frame, the electron-ion collision operator, accurate to first order in $(1 / x)$ and including pitch-angle scattering and energy diffusion can be expressed as,

$$
C_{e i}\left(\delta f^{e}\right)=\nu_{0} \frac{1}{2} \frac{\partial}{\partial \xi}\left(1-\xi^{2}\right) \frac{\partial}{\partial \xi}+\nu_{0} v \frac{\partial}{\partial v}\left(\frac{m_{e}}{m_{i}} \delta f^{e}+\frac{v_{t h i}^{2}}{2 v} \frac{\partial}{\partial v} \delta f^{e}\right) .
$$

For the present analysis, only the pitch angle scattering needs to be retained.

Ion-electron collisions can be simply modeled as ion Brownian motion in an electron fluid. Only the friction force by the electrons must be retained since the ion-ion collisions provide the ion-velocity-space diffusion on a time scale much faster than that of the ion-electron collisions. The local momentum loss of the electrons due to electron-ion collision is properly taken into account, and the first order ion momentum is then modified to ensure that the local momentum conservation between ions and electrons is maintained.

\section{TWO SPECIES SIMULATIONS}

\section{A. Role of Like-Species Collisions}

To the lowest order in the mass ratio expansion, the ion collision operat,or only includes ion-ion collisions. It is well known that due to the momentum conservation like-species collisions alone should produce no neoclassical particle transport. It is demonstrated in Fig. 10 that ion particle 
fluxes resulting from like-species collisions will indeed drop to zero when appropriate conservation properties are retained in the simulations. In the case of no temperature gradient, $\kappa_{t}=0$, the energy flux is also expected to vanish. ${ }^{1}$ In Fig. 11 , the new collision operator developed in Section IV gives the correct zero energy flux, while the Dimits and Cohen model produces an unphysical inward energy flux. We note that this is the first dynamic simulation which clearly verify the zero flux result for like-species collisions. The total $f$ scheme has considerable difficulty in implementing kinetic collision operators with all conservation properties retained. Although attempts have been made to implement binary collisions including all conservation properties for total $f$ schemes, ${ }^{10}$ definitive results of the type reported here have not been published. Ion-ion collisions are known to produce a toroidal neoclassical rotation through the decay of poloidal rotation due to magnetic pumping. ${ }^{22}$ Although this parallel flow is present, momentum conservation prevents collisions from generating frictional forces. As a result, since pressure variations or stress anisotropy cannot be set up, and no flux is induced because of the absence of outward diamagnetic-type drifts. A more important consequence of ion-ion collisions is the enhanced ion energy flux in the presence of a temperature gradient. Since this flux is a square root of the mass ratio larger than that of the electron, they can dominate ion thermal transport under certain circumstance. In Fig. 12 the dependence of ion energy fluxes on the effective collision frequency is plotted, and a comparison of the simulations results to the theoretical results of Hinton and Hazeltine 1 is illustrated.

Complete electron dynamics requires including electron-electron collisions as well as electron-ion collisions since they are on the same time scale. To show the importance of conservation properties, we compare results from an electron-electron collision model conserving momentum and energy with a non-conserving model. In Figs. 13 and 14, the results of particle flux and bootstrap current are compared with theoretical predictions from Hinton and Hazeltine's review paper (with $T_{i}=0$ ).$^{1}$ Here it is seen that the momentum-conserving collision operators give a much better fit to the theoretical calculation results in the entire range of collisionality. Furthermore, as predicted, the electron-electron collisional contribution to neoclassical particle transport only occurs in the presence of electron-ion collisions. Estimates for the particle flux from the non-conserving collision model overestimates the neoclassical flux in the collisiona' regime. The reason is that the flux here is caused by parallel frictional forces due to first order parallel flows. 
Since the electron-electron collisions do not contribute to this frictional force in lowest order because of momentum conservation, the associated flux is negligible. On the other hand, the test particle drag and diffusion operators without momentum conservation tend to drive the distribution function to a Maxwellian and thus artificially create frictional forces. In the banana regime, the particle fluxes are dominated by trapping and de-trapping processes. Thus only the test particle drag and diffusion terms are important. For the bootstrap current, the operators without momentum conservation underestimate the current. This is because the bootstrap current is governed by the momentum balance of circulating electrons. The electron-electron collisions should contribute to the rate of momentum transfer from trapped particles to circulating particles but not to that from electron to ions. Again, the operators without momentum conservation create an artificial frictional force and therefore cause additional unrealistic momentum loss of circulating electrons to ions. Consequently, the results from such collision operators fit quite well only with the theoretical results of $Z_{\text {eff }}=\infty$, where electronelectron collision can be neglected.

\section{B. Ambipolarity}

Ion dynamics affects electron transport through the collisional coupling and quasi-neutrality constrain. When ion dynamics is retained in twospecies simulations with $T_{i}=T_{e}$ and $\kappa_{t}=0$, the bootstrap current as well as electron particle and energy fluxes are doubled with respect to the single-species simulation results (corresponding to $T_{i}=0$ ) (as expected from neoclassical theory).

For a simple plasma, ion and electron particle fluxes are automatically ambipolar because of the momentum conservation between ions and electrons. This key ambipolar feature is demonstrated for the first time in the present dynamical simulations. Representative results are displayed in Fig. 15.

\section{TOROIDAL FLOWS}

\section{A. Basic Formalism}

In steady state, toroidal flow is a function only of the magnetic surface; i.e., $\mathbf{u}_{0}=u(r) \hat{\mathbf{e}}_{\mathrm{t}}=\omega(r) R_{0} \hat{\mathbf{e}}_{\mathrm{t}}$. Following the approach of Hinton and Wong, ${ }^{17}$ 
we transform to a rotating frame, $\mathbf{v}-\mathbf{u}_{\mathbf{0}} \rightarrow \mathbf{v}$, linearize and then gyroaverage the Fokker-Planck equation for the ion distribution function. The inertial forces, and thus the guiding center drifts associated with these forces, are retained in this rotating frame. The governing linearized drift kinetic equation becomes,

$$
v_{\|} \hat{\mathbf{b}} \cdot \nabla \delta f-C(\delta f)=v_{d r}\left(\kappa_{n}+\left(\frac{m \varepsilon}{T}-\frac{3}{2}\right) \kappa_{t}+\frac{m}{T} \omega R\left(v_{\|}+\omega R\right) \kappa_{\omega}\right) F_{0},
$$

where $\kappa_{\omega}$ is the inverse of angular velocity scale length. Here the zeroth order distribution function is,

$$
F_{0}=n_{0}(r) F_{m}=\frac{n_{0}(r)}{\pi^{\frac{3}{2}} v_{t h}^{3}} \exp \left(-\frac{\varepsilon}{T(r)}\right)
$$

where the invariants of motion are defined in the rotating frame,

$$
\mu=\frac{v_{\perp}^{2}}{2 B}
$$

and

$$
\varepsilon=\frac{1}{2}\left(v_{\|}^{2}+v_{\perp}^{2}\right)+\frac{e}{m} \Phi-\frac{\omega^{2} R^{2}}{2} .
$$

The guiding center drift velocity includes the usual $\nabla B, \nabla \times \mathbf{B}$ and $\nabla \Phi \times \mathbf{B}$ drift terms together with new drift terms produced by the centrifugal force, $m \omega^{2} \mathbf{R}$, and Coriolis force, $2 m v_{\|} \hat{\mathbf{b}} \times \omega \hat{\mathbf{e}}$, in the rotating frame. This has the form:

$$
\mathbf{v}_{\mathbf{d}}=\frac{\hat{\mathbf{b}}}{\Omega} \times\left(\frac{v_{\perp}^{2}}{2} \frac{\nabla B}{B}+v_{\|}^{2} \hat{\mathbf{b}} \cdot \nabla \hat{\mathbf{b}}+\frac{e}{m} \nabla \Phi-\omega^{2} \mathbf{R}+2 \omega \hat{\mathbf{e}_{\mathbf{z}}} \times \hat{\mathbf{b}} v_{\|}\right) .
$$

The electrostatic potential is defined solely by charge neutrality, and can be represented as;

$$
e \Phi=\frac{T_{e}}{T_{e}+T_{i}} \frac{m_{i} \omega^{2}}{2}\left(R^{2}-<R^{2}>\right)
$$

\section{B. Effects of Toroidal Flows without Electrostatic Field}

To separate the effects of the poloidal electrostatic field and rotation, one approach is to set this potential to zero and assume charge neutrality

is achieved by other mechanisms. The solution of Eq. (20) gives rise to 
neoclassical viscosity $\left(\mu_{i}\right)$ and ion thermal conductivity $\left(\chi_{i}\right)$. In the large aspect ratio limit of the banana regime, the results are, ${ }^{17}$

$$
\mu_{i}=0.1 \frac{q^{2} \rho_{i}^{2}}{\tau_{i}}
$$

and

$$
\chi_{i}=\frac{0.66 q^{2}}{\epsilon^{1.5}} \frac{\rho_{i}^{2}}{\tau_{i}}\left(1+2.24 \zeta-3.62 \zeta^{2}+2.32 \zeta^{3}\right)
$$

where $\zeta$ is defined by $\zeta \equiv m_{i} \omega^{2} R^{2} / 2\left(T_{i}+T_{e}\right)$.

The radial derivative of the angular velocity appear in the linearized drift kinetic equation as a driving term. Therefore, Eq. 20 is a generalization of the usual linearized drift kinetic equation, Eq. 3, and can be readily solved by the numerical scheme developed in Section II.

Simulation results including toroidal viscosity are shown in Fig. 16. They agree quite well with the theoretical results of Hinton and Wong. ${ }^{17}$ Specifically, it is confirmed that the neoclassical enhancement of the viscosity is a Pfirsch-Schluter factor times the classical viscosity in banana regime and that there is no enhancement in the collisional regime. No anomalous viscosity is observed. It is noted that if energy conservation property of the collision operator is not incorporated, results indicate a much higher viscosity.

Fig. 17 shows the toroidal mass flow enhancement of ion energy fluxes in the banana regime, in agreement with Eq. 23. This enhancement comes from the additional guiding center drift associated with the centrifugal force. It is observed in these two species simulations that when the Mach number is much smaller than unity, the influence of toroidal fiow on electron transport and the bootstrap current is negligible.

\section{Effects of Electrostatic Field Associated with Toroidal Flows}

The effects of the electrostatic field associated with the toroidal flows can be included by adding the equilibrium potential defined in Eq.(21). It is found that this electric field can enhance both the density gradient driven electron particle flux and the bootstrap current, but tend to reduce temperature gradient driven electron particle flux and the associated bootstrap current. The effect on electron thermal fluxes again tends to be negligibly small for small Mach number. This behavior is likely associated with the 
fact that the poloidal electric field has a stronger influence(trapping and de-trapping) on low energy particles than on those at high energies.

\section{NEOCLASSICAL TRANSPORT FOR STEEP GRA- DIENT PROFILE}

The standard neoclassical theory assumes that the ion poloidal gyroradius is much smaller than the equilibrium profile scale length $\left(\rho_{p} \ll L_{p}\right)$. However, this assumption breaks down both in the tokamak edge regime, where steep gradient profiles have been observed in the $\mathrm{H}$ mode plasmas, and in the region close to the seperatrix of diverted tokamaks. This motivates analysis of possible new physics effects when the usual small gyroradius ordering is not invoked. Using the formalism developed in Section II, both numerical and analytical studies have been carried out to investigate the finite banana width corrections to standard neoclassical theory. Simulation results indicate that the ion thermal flux and toroidal mass flow increase due to the finite banana orbit size. As shown in Fig. 18, simulation results show that the ion thermal flux increases linearly with $\left(\rho_{p} \kappa\right)^{2}$. In order to analytically estimate the finite orbit size correction, we adopt the smallness ordering $\left(\rho_{p}<L_{p}\right)$. The drift kinetic equation based on this small parameter is then expanded with the lowest order correction retained.

\section{A. Enhancement of Ion Thermal Flux}

For a qualitative estimate of the ion thermal flux, we consider the following steady state drift kinetic equation,

$$
v_{\|} \hat{\mathbf{b}} \cdot \nabla f_{1}+\mathbf{v}_{\mathbf{d}} \cdot \nabla f_{1}-C\left(f_{1}\right)=-\mathbf{v}_{\mathbf{d}} \cdot \nabla F_{m} .
$$

A simplified Lorentz model is employed in the banana regime; i.e.,

$$
C=\nu 2 h \xi \frac{\partial}{\partial \lambda} \lambda \xi \frac{\partial}{\partial \lambda} \text {. }
$$

Expanding $f_{1}$ using banana ordering, the zeroth order equation becomes,

$$
v_{\|} \hat{\mathbf{b}} \cdot \nabla f_{1}^{(0)}+\mathbf{v}_{\mathbf{d}} \cdot \nabla f_{1}^{(0)}=-\mathbf{v}_{\mathbf{d}} \cdot \nabla F_{m} .
$$

Only the radial drift velocity needs to be retained here since the poloidal component make a neglecgible contribution to the banana size. Hence,

$$
v_{\|} \hat{\mathbf{b}} \cdot \nabla\left(f_{1}^{(0)}-\frac{v \kappa}{\Omega_{p}} h \xi\right) F_{m}=-\frac{v_{\|}}{\Omega_{p}} \hat{\mathbf{b}} \cdot \nabla\left(h v_{\|}\right) \frac{\partial f_{1}^{(0)}}{\partial r} .
$$


This equation can be solved iteratively by treating the term on the right side as a small perturbation; i.e.,

$$
f_{1}^{(0)}=\left(1+\frac{v \kappa}{\Omega_{p}} h \xi\right) \frac{v \kappa}{\Omega_{p}} F_{m}\left(h \xi+\bar{f}_{1}\right),
$$

where

$$
\frac{\partial \bar{f}_{1}}{\partial \theta}=0
$$

We then apply the annihilator,

$$
\oint \frac{d \theta}{v_{\|}}
$$

to the first order equation

$$
v_{\|} \hat{\mathbf{b}} \cdot \nabla f_{1}^{(1)}+\frac{v_{\|}}{\Omega_{p}} \hat{\mathbf{b}} \cdot \nabla\left(h v_{\|}\right) \frac{\partial f_{1}^{(1)}}{\partial r}-C\left(f_{1}^{(0)}\right)=0 .
$$

Again, to simplify the orbit averaged collision operator, we employ the iterative scheme.

$$
\frac{\partial \bar{f}_{1}}{\partial \lambda}=\frac{v \kappa}{\Omega_{p}} \frac{\langle h \xi\rangle}{\langle\xi\rangle}+\frac{1}{2\langle\xi\rangle} H\left(h_{\min }-\lambda\right),
$$

where $\mathrm{H}$ is a simple model step function. The next order contribution from the drift term is proportional to $\left(\rho_{p} \kappa\right)^{2}$. Thus, the distribution function exact to first order is given by:

$$
f_{1}^{0}=\left(1+\frac{v \kappa}{\Omega_{p}} h \xi\right) \frac{v \kappa}{\Omega_{p}} F_{m}\left[h \xi+\frac{v \kappa}{\Omega_{p}} \frac{\langle h \xi\rangle}{\langle\xi\rangle}+\frac{1}{2<\xi>} H\left(h_{\min }-\lambda\right)\right]
$$

Note that the first order correction does not contribute to the ion thermal flux, while the second order correction makes a positive contribution. Specifically,

$$
Q=<\int d^{3} v \frac{1}{2} m v^{2} v_{d r} f_{1}>=Q_{0}\left(1+\alpha\left(\rho_{p} \kappa\right)^{2}\right)
$$

Here $Q_{0}$ is the usual neoclassical thermal flux with zero orbit size and $\alpha$ is a positive number. This simple estimate confirms the same trend as that observed in the simulation; i.e., ion thermal flow is increased by a factor of order $\left(\rho_{p} \kappa\right)^{2}$. 


\section{B. Density-Gradient-Driven Neoclassical Poloidal Rotation}

Using the fact that the ion-ion collision operator annihilates a shifted Maxwellian, we can solve Eq. (3) when temperature is uniform $\left(\kappa_{t}=0\right)$. This yields

$$
f_{1}^{(0)}=\left[1+\left(\frac{v \kappa}{\Omega_{p}} h \xi\right)^{2}\right] \frac{v \kappa}{\Omega_{p}} F_{m} h \xi .
$$

It then follows that the associated parallel mass flow is increased from the usual neoclassical toroidal flow by a factor of $\left[1+\left(\rho_{p} \kappa\right)^{2}\right]$. As a consequence, the poloidal component of the parallel flow no longer balances the ion diamagnetic flow. A net poloidal rotation is thereby generated. If the impurity density gradient is the same as that of the main ion component, this effect should be stronger because of the larger gyroradius.

\section{CONCLUSIONS}

A gyrokinetic simulation of steady state, multi-species neoclassical transport has been successfully carried out for the first time. Simulation results using appropriate model collision operators are found to agree very well with standard neoclassical theory.

A new $\delta f$ scheme to deal with this class of problem has been developed and implemented including appropriate collision operators conserving momentum and energy. The importance of momentum and energy conservation is demonstrated; i.e. it is shown that significant qualitative errors are introduced if the conservation properties are violated.

Ion dynamics are self-consistently retained for the first time in a multispecies simulation, and it is dynamically demonstrated that: (i) like-species collisions produces no particle flux, and (ii) neoclassical fluxes are automatically ambipolar for a simple ion-electron plasma.

Toroidal flow has also been introduced for the first time into these simulations. Trend from the neoclassical viscosity theory of Hinton and Wong is confirmed. The poloidal electric field associated with this flow is found to enhance the density gradient driven electron particle flux and the bootstrap current.

Neoclassical theory appropriate for steep equilibrium gradient profiles is examined both analytically and numerically. It is shown that both the ion thermal flux and the toroidal mass flow increase by factors of $\left[1+(\rho \kappa)^{2}\right]$ when the finite banana width effects are taken into account. 
Building on the advantages of the present approach, (e.g., multi-species capability, steady state, fully dynamical approach, low noise), we will explore in future studies potential significant modifications of neoclassical transport which are usually inaccessible by conventional calculation processes. These investigations will deal with sheared toroidal flows, energetic particle physics, fluctuating fields(non-self-consistent and self-consistent), and realistic geometric effects in advanced tokamaks and stellarators.

\section{ACKNOWLEDGMENTS}

This work was supported by the U.S. Department of Energy under Contract No. DE-AC02-76-CHO3073. The authors would like to thank Dr. S. E. Parker for useful discussion and for helping us the initial coding. 


\section{References}

${ }^{1}$ F. L. Hinton and R. D. Hazeltine, Rev. Mod. Phys. 48, 239 (1976).

${ }^{2}$ K. C. Shaing, Phys. Fluids B 2, 764 (1990).

${ }^{3}$ K. C. Shaing and R. D. Hazetine, Phys. Fluids B 2, 2353 (1990).

${ }^{4}$ T. E. Stringer, Nucl. Fusion 32, 1421 (1992).

${ }^{5}$ A. H. Boozer and H. J. Gardner, Phys. Fluids B 2; 2408 (1990).

${ }^{6}$ R. Iacono and A. Bhattacharjee, Phys. Fluids B 4, 1685 (1992).

${ }^{7}$ H. Maassberg, R. Burhenn, U. Gasparino, G. Kuhner, and H. Ringler, Phys. Fluids B 5, 3627 (1993).

${ }^{8}$ K. T. Tsang, Y. Matsuda, and H. Okuda, Phys. Fluids 18, 1282 (1975).

${ }^{9}$ Y. Wu and R. B. White, Phys. Fluids B 5, 3291 (1993).

${ }^{10}$ S. Ma, R. Sydora, and J. Dawson, Bull. Am. Phys. Soc. 37, 1546 (1992).

${ }^{11}$ W. W. Lee, J. Comput. Phys. 72, 243 (1987).

${ }^{12}$ A. M. Dimits and W. W. Lee, J. Comput. Phys. 107, 309 (1993).

${ }^{13}$ S. E. Parker and W. W. Lee, Phys. Fluids B 5, 77 (1993).

${ }^{14}$ A. Y. Aydemir, 1993, private communication.

${ }^{15}$ G. Hu and J. A. Krommes, Phys. Plasma 1, 862 (1994).

${ }^{16}$ A. M. Dimits and B. I. Cohen, Phys. Rev. E 49, 709 (1994).

${ }^{17}$ F. L. Hinton and S. K. Wong, Phys. Fluids 28, 3082 (1985).

${ }^{18}$ X. Q. Xu and M. N. Rosenbluth, Phys. Fluids B 3, 627 (1991).

${ }^{19}$ B. A. Trubnikov, Review of Plasma Physics, volume 1, Consultants Bureau, New York, 1965.

${ }^{20}$ J. L. Spitzer, Physics of Fully Ionized Gases, Interscience, New York, 1967.

${ }^{21}$ T. D. Rognlien and T. A. Cutler, Nucl. Fusion 20, 1003 (1980).

${ }^{22}$ T. H. Stix, Phys. Fluids 16, 1260 (1973). 


\section{FIGURE CAPTIONS}

FIG. 1. Particle flux $\Gamma$ versus $\nu^{*}$ computed using simplified Lorentz collision model. The solid line is the analytical neoclassical result. .

FIG. 2. Energy flux $Q$ versus $\nu^{*}$ computed using simplified Lorentz collision model. The solid line is the analytical neoclassical result.

FIG. 3. Bootstrap current $j_{b}$ (normalized by $j_{0}$ ) versus $\nu^{*}$ computed using simplified Lorentz collision model.

FIG. 4. Poloidal variations of $\delta n(\theta) / n_{0}$ and $u_{1}(\theta) / v_{t h}$ in the collisional regime.

FIG. 5. Time history of particle flux in the collisional regime.

FIG. 6. Time history of bootstrap current in the banana regime.

FIG. 7. Particle fluxes $\Gamma$ versus $\nu^{*}$ for the Lorentz model $(x)$ and pitch-angle scattering model of Hinton and Hazeltine $(+)$. The solid line is theoretical the analytical result of Hinton and Hazeltine (1976).

FIG. 8. Comparison of collision operators. Dashed line represents linearized shifted Maxwellian, solid line represents results using model improved operator, and dotted line represents results using Dimits-Cohen operator.

FIG. 9. Time history of the third velocity moment. Solid line represents results using improved operator, and the dotted line represents results DimitsCohen operator.

FIG. 10. Ion particle flux time history (time averaging) resulting only from ion-ion collisions.

FIG. 11. Ion energy flux time history (time averaging) due only to ion-ion collisions $\left(\kappa_{t}=0\right)$. Solid line represents results using improved operator, and the dotted line represents results Dimits-Cohen operator.

FIG. 12. Ion energy fluxes $Q$ versus $\nu^{*}\left(\kappa_{t} \neq 0\right)$. The dashed line is the analytical neoclassical result.

FIG. 13. Particle fluxes $\Gamma$ versus $\nu^{*}$ for ion-electron and electron-electron collisions with momentum conservation $(x)$ and without momentum conservation $(+)$. The solid line represents the analytical result of Hinton and Hazeltine (1976) for $Z_{\text {eff }}=1$. 
FIG. 14. Bootstrap current $j_{b}$ (normalized by $j_{0}$ ) versus $\nu^{*}$ with ion-electron and electron-electron collisions with momentum conservation $(x)$ and without momentum conservation( + ). The solid line represents the analytical result for $Z_{\text {eff }}=\infty$. The dashed line corresponds to $Z_{\text {eff }}=1$.

FIG. 15. Time history of Ambipolar particle fluxes (time averaged). Solid line is for the ion, and dashed line is for the electron.

FIG. 16. Ion momentum flux versus $\nu^{*}$ normalized by analytical result in collisionless limit.

FIG. 17. Ion energy fluxes $Q$ versus $\xi$ normalized with the corresponding value in the zero rotation limit. Solid line represents the analytical results.

FIG. 18. Increasing of ion energy flux with the parameter $\rho \kappa$. Here $\delta Q=$ $Q-Q_{0}$, and $Q_{0}$ is the ion flux in the limit of $\rho \kappa=0$ 


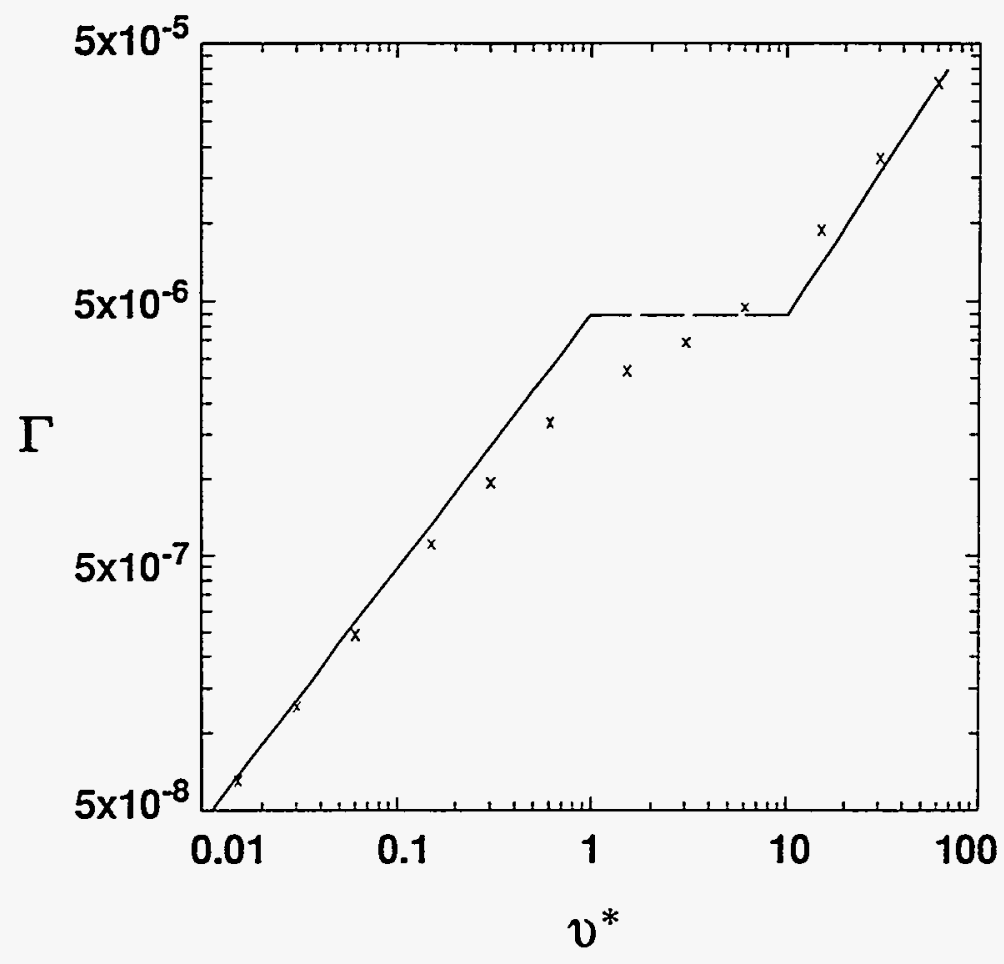

Figure 1: 


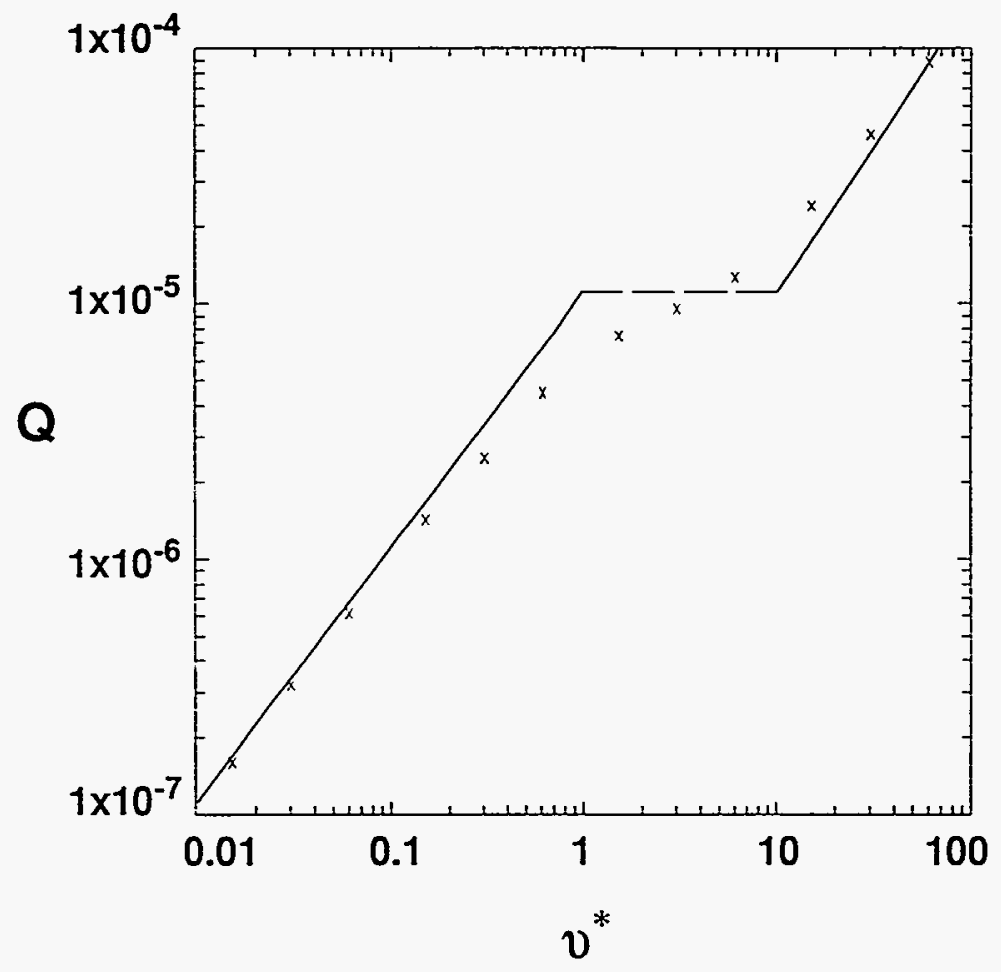

Figure 2: 


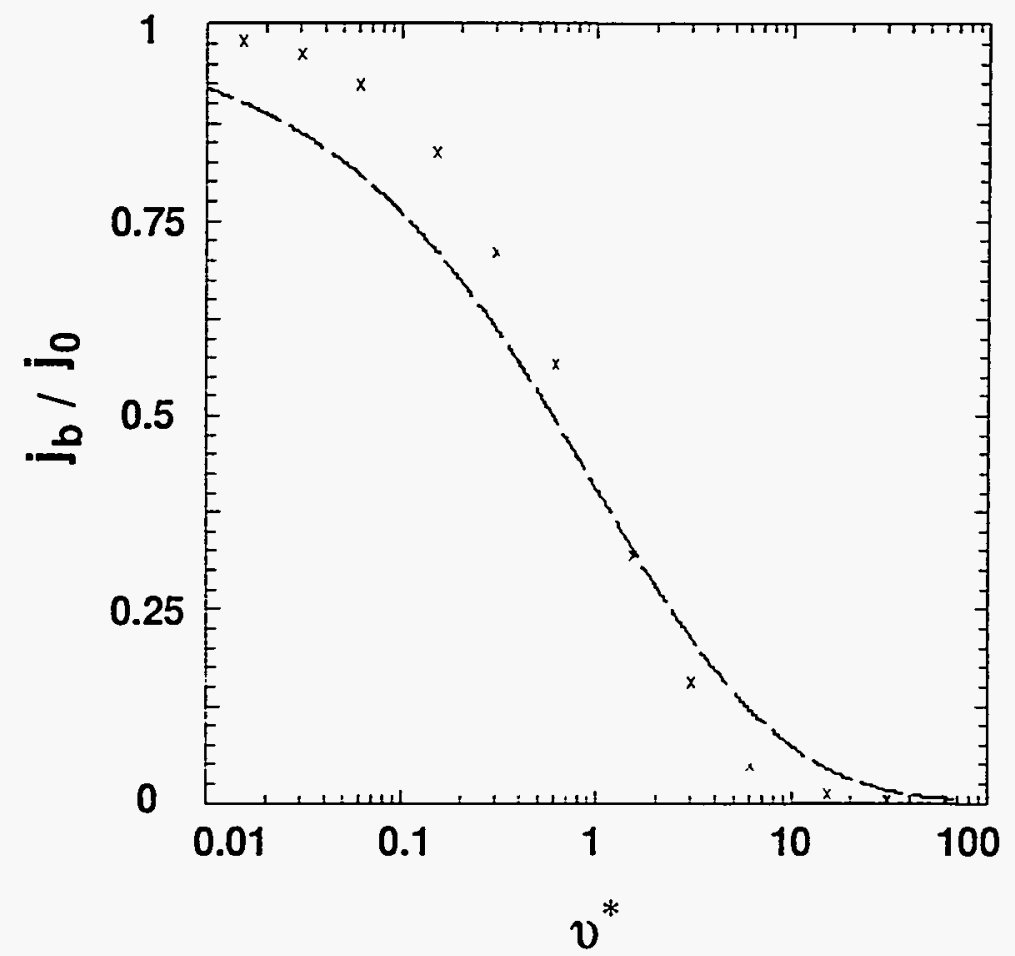

Figure 3: 


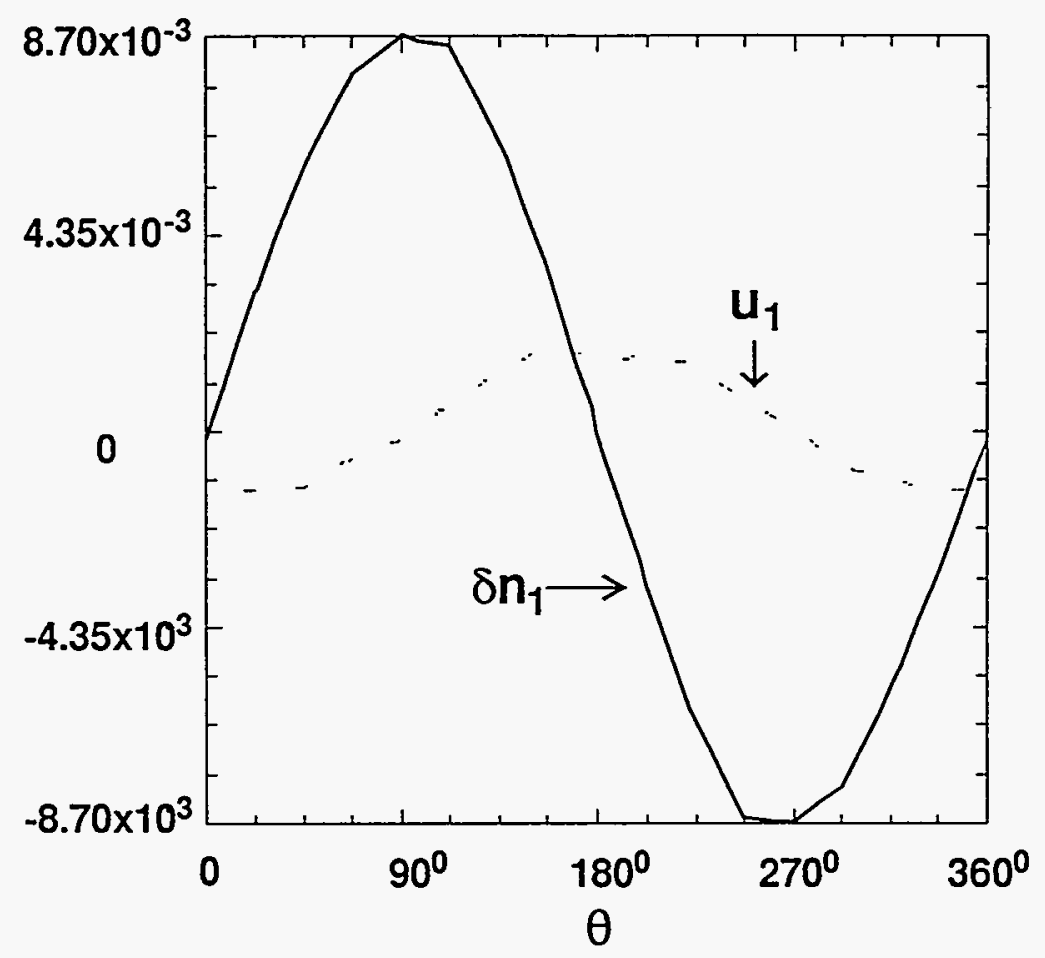

Figure 4: 


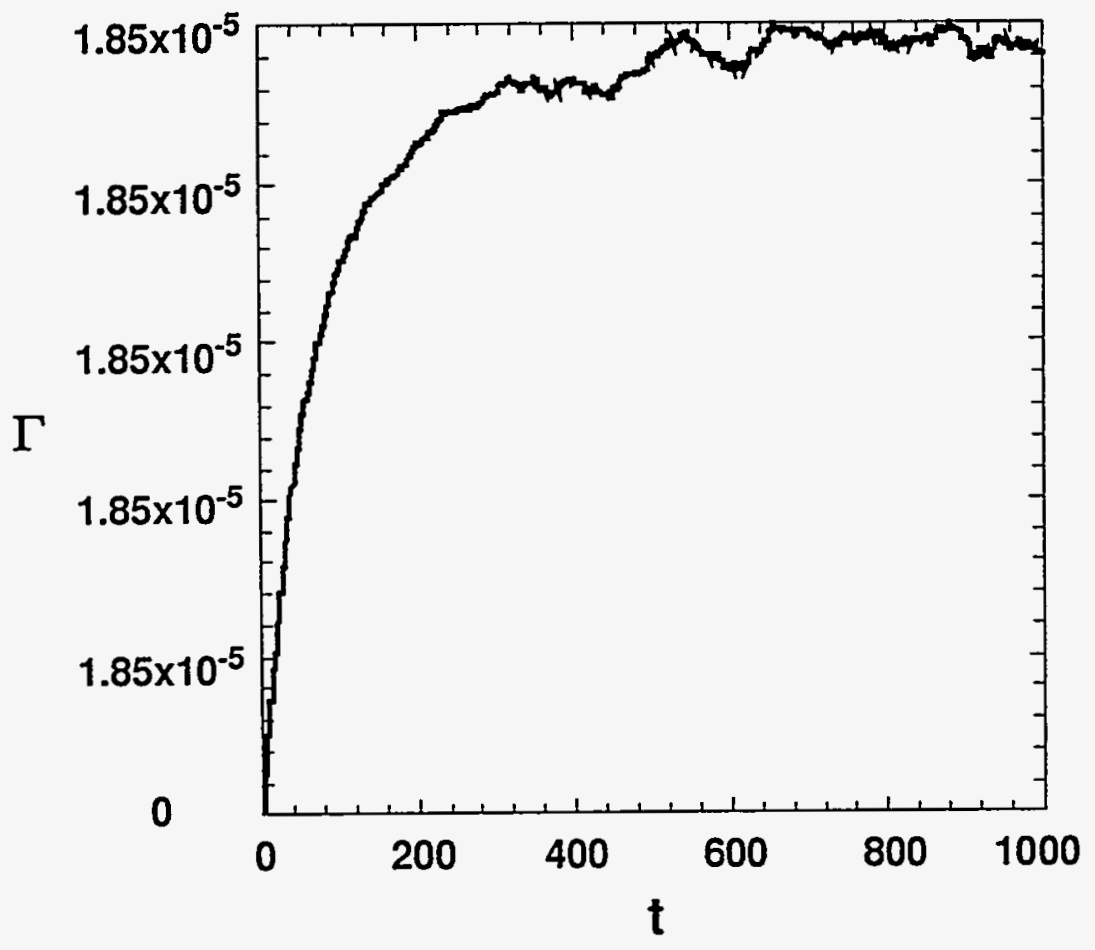

Figure 5: 


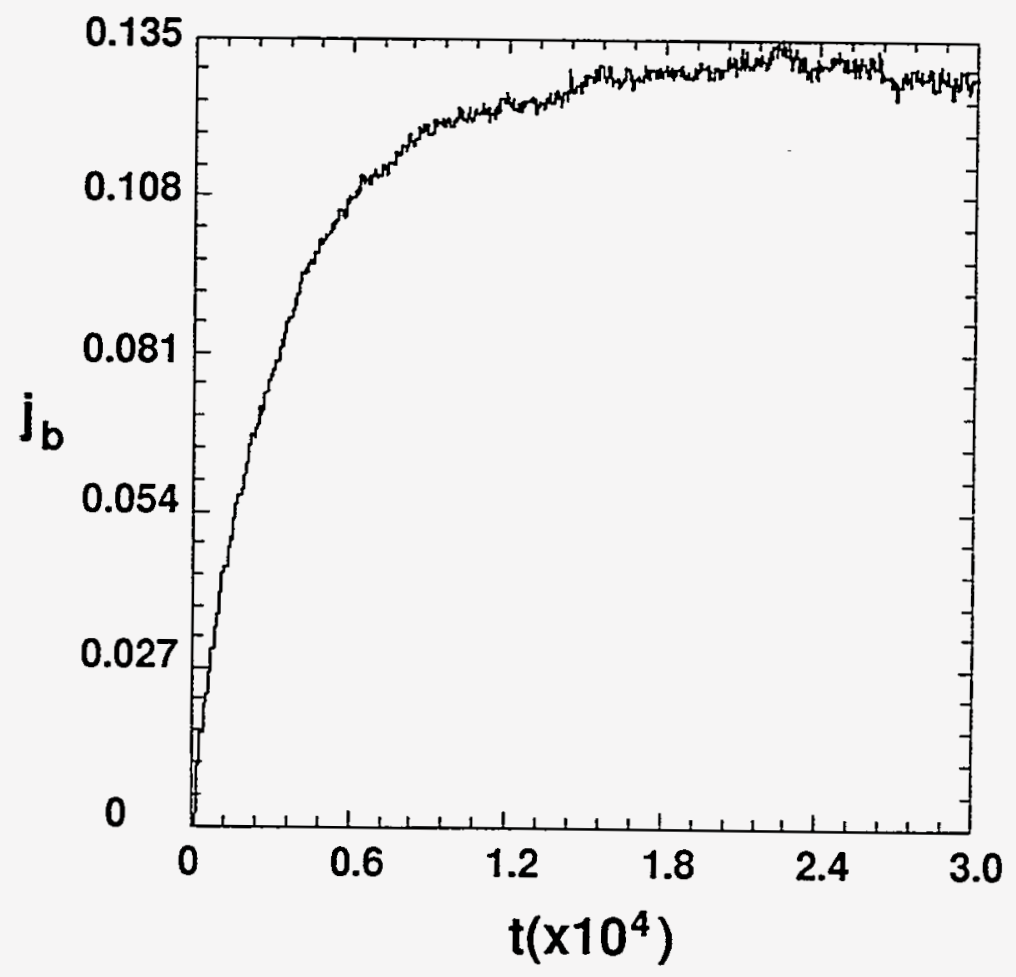

Figure 6: 


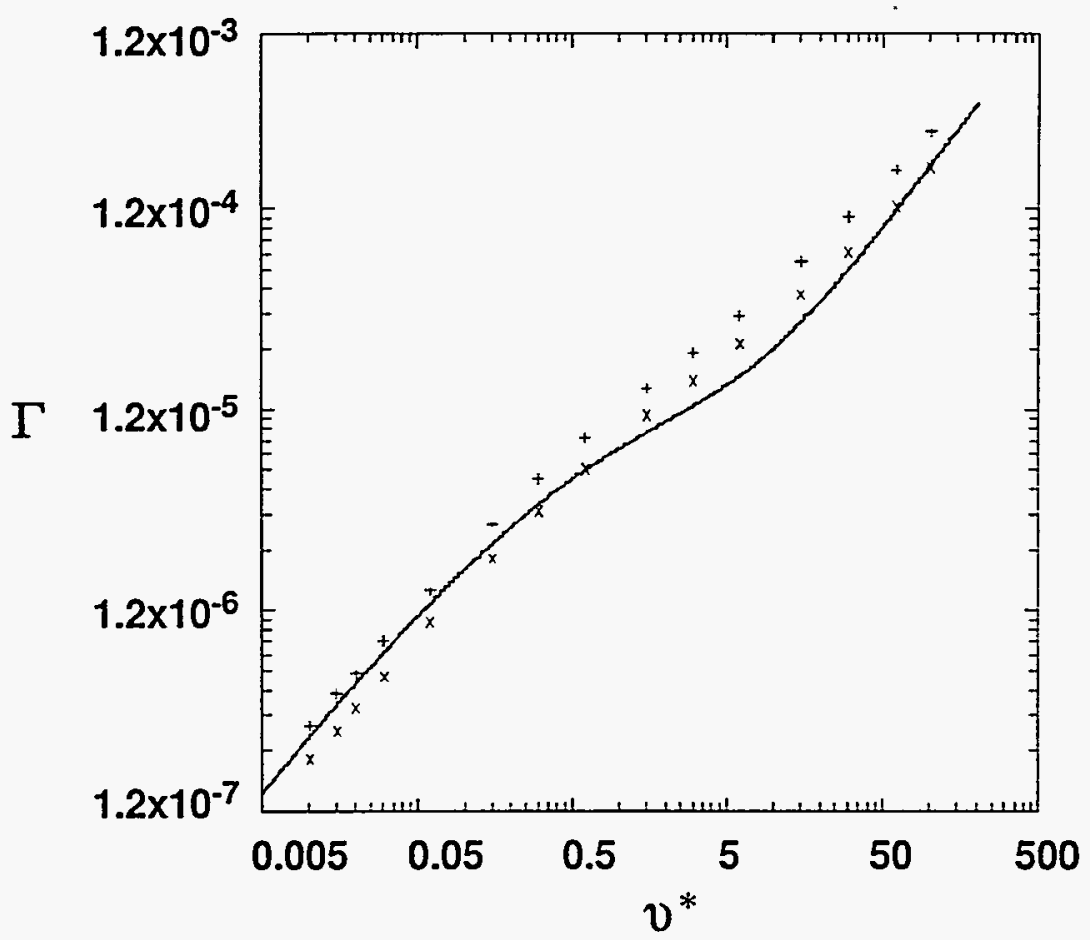

Figure 7: 


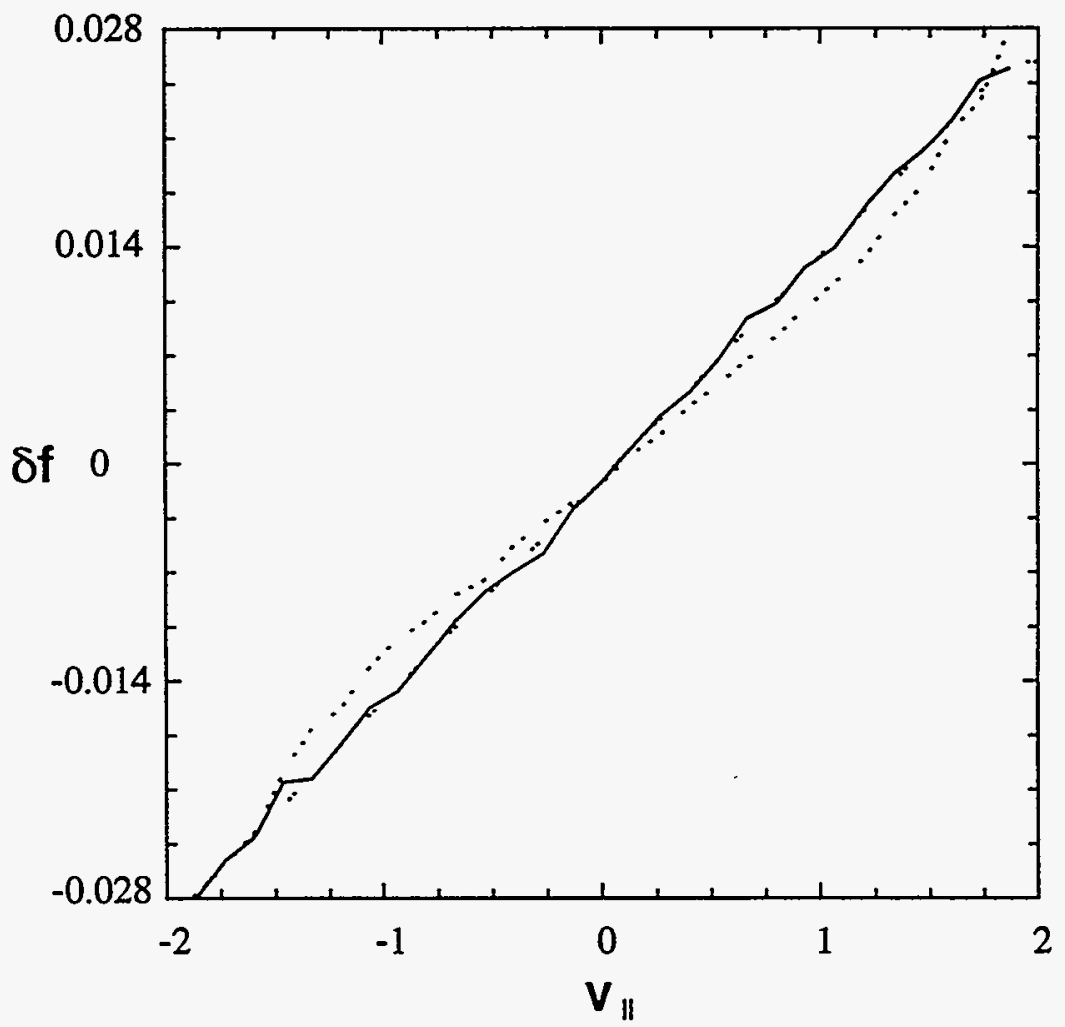

Figure 8: 


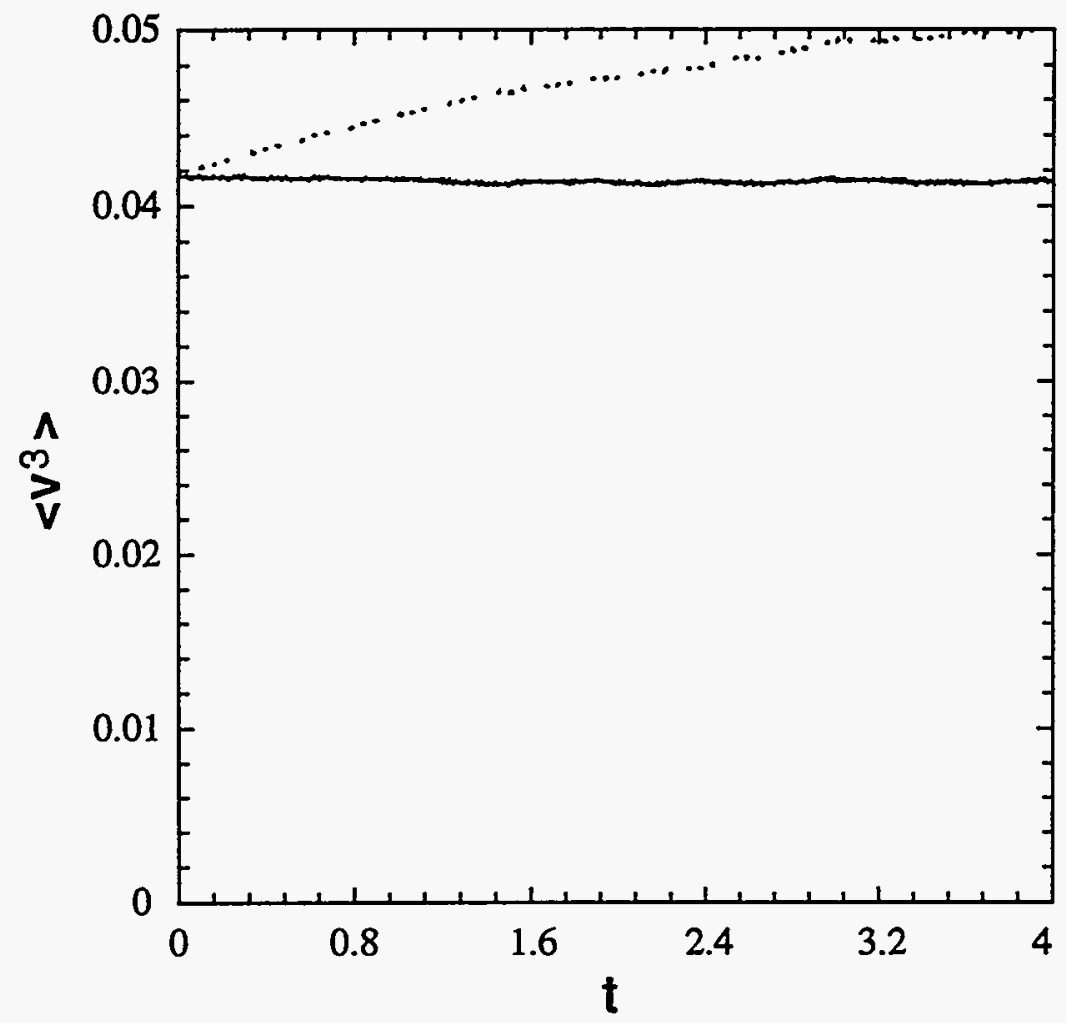

Figure 9: 


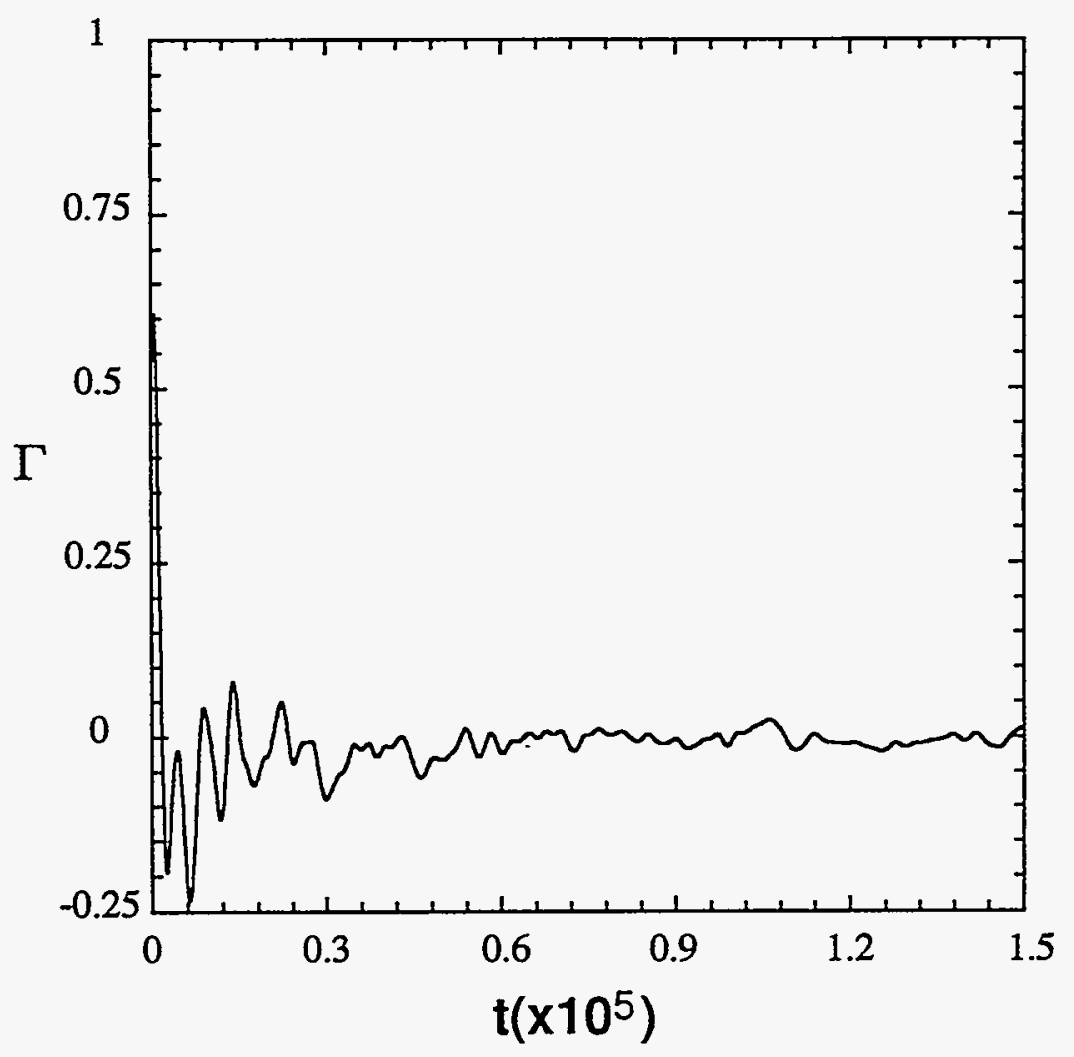

Figure 10: 


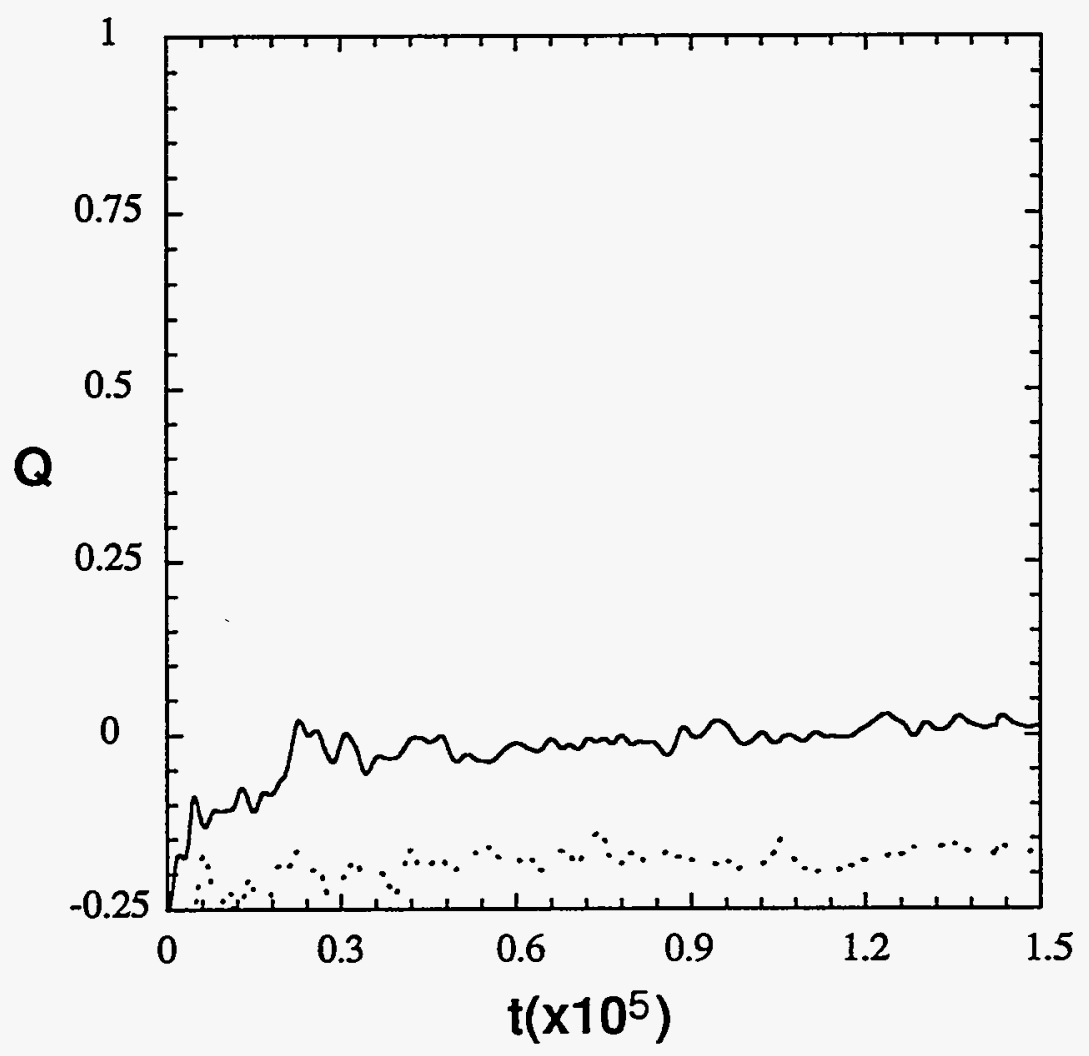

Figure 11: 


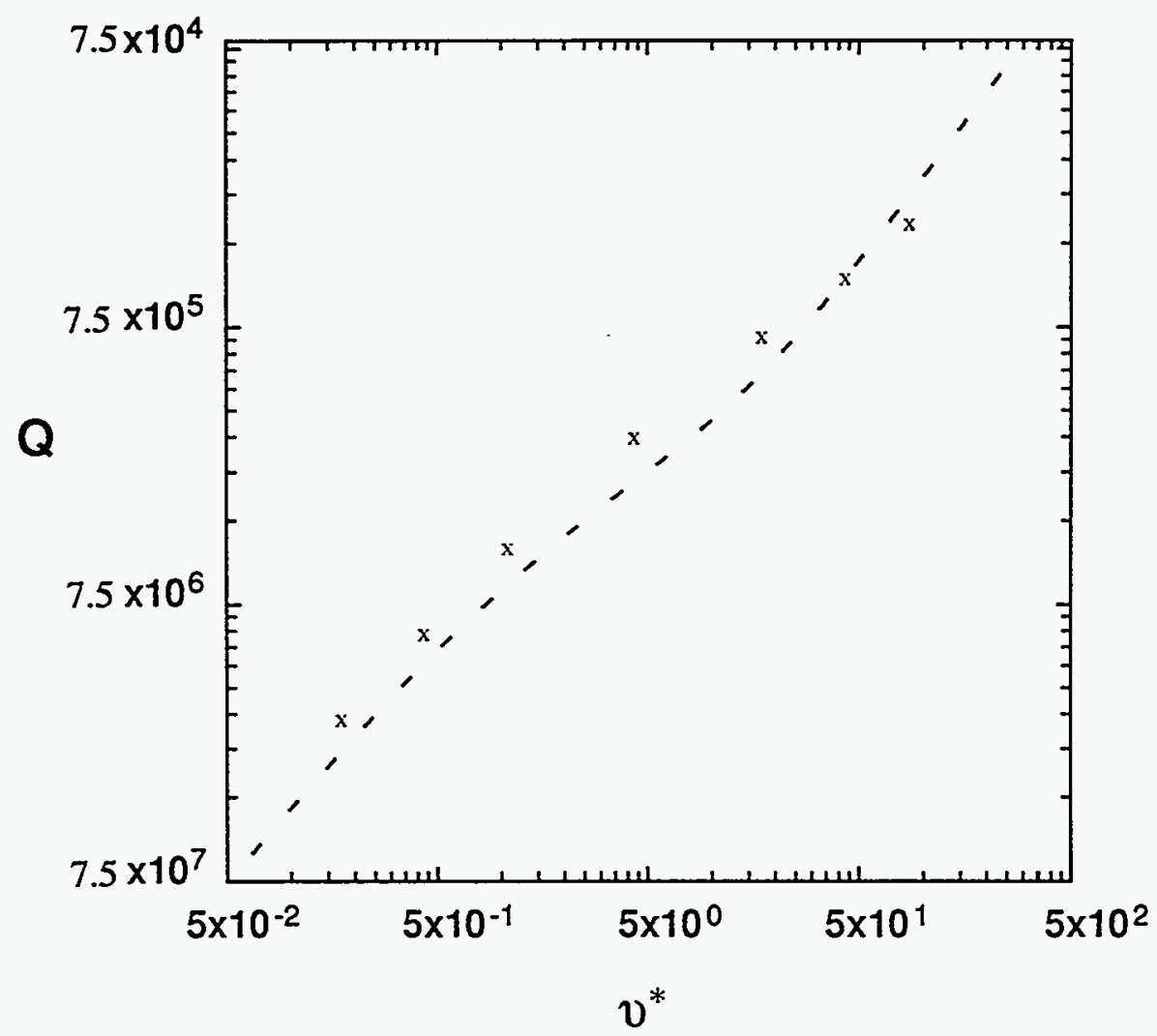

Figure 12: 


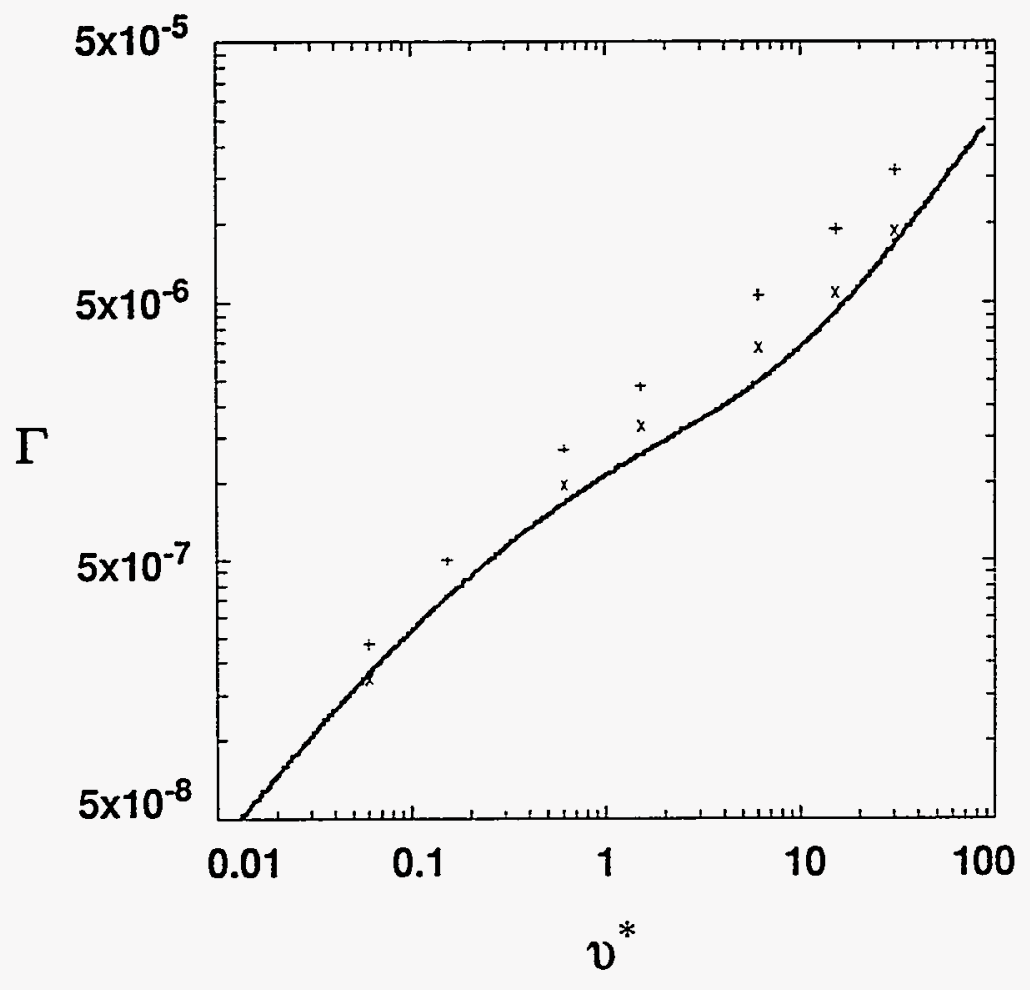

Figure 13: 


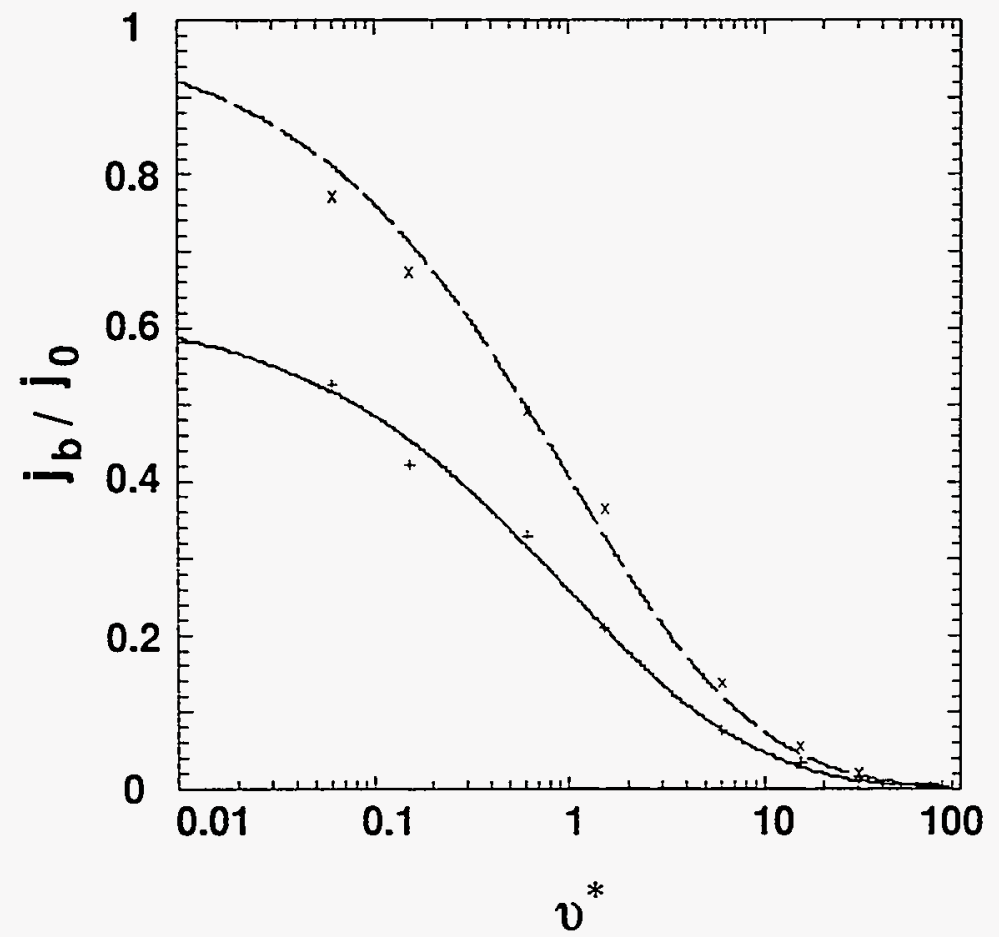

Figure 14: 


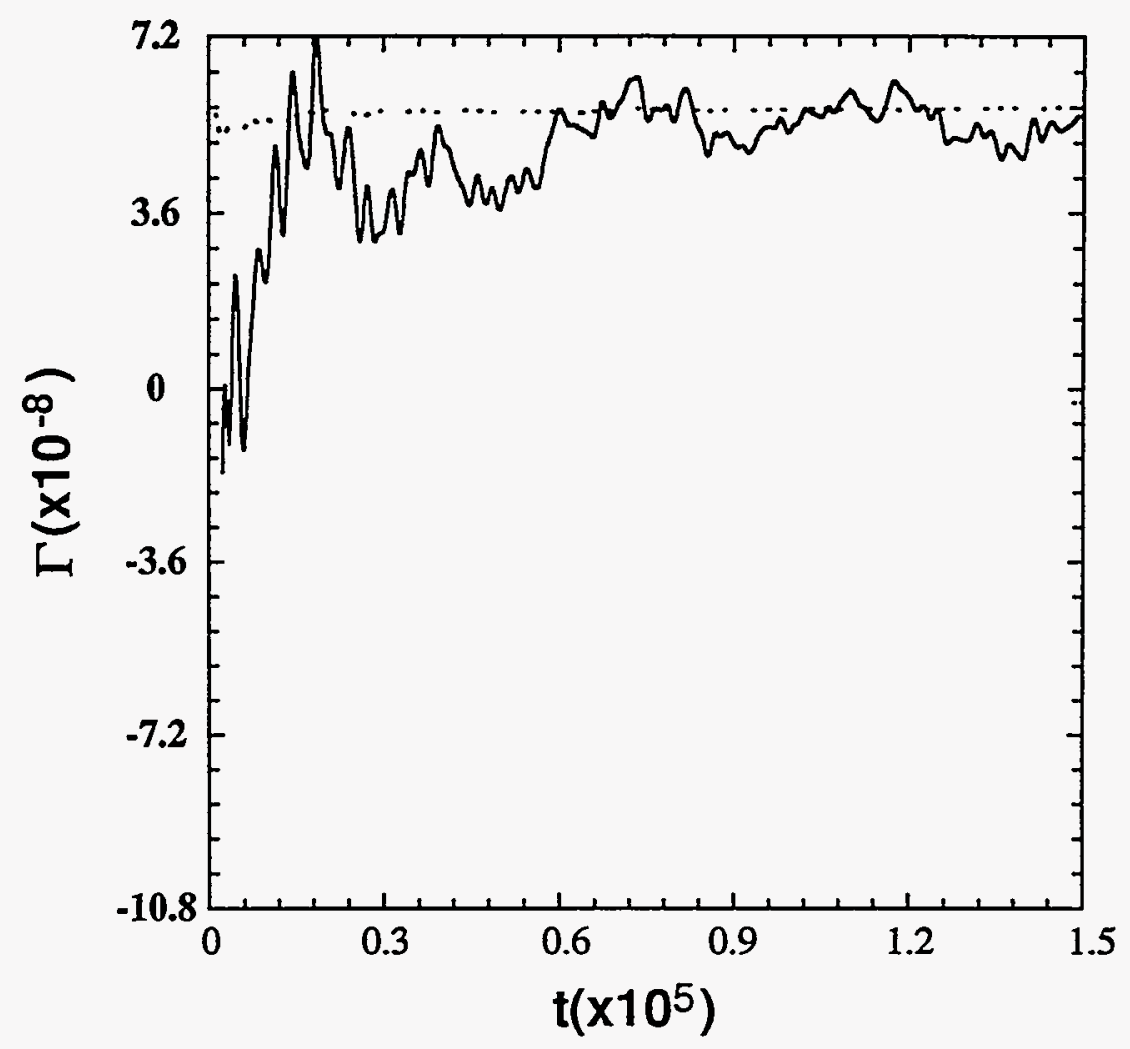

Figure 15: 


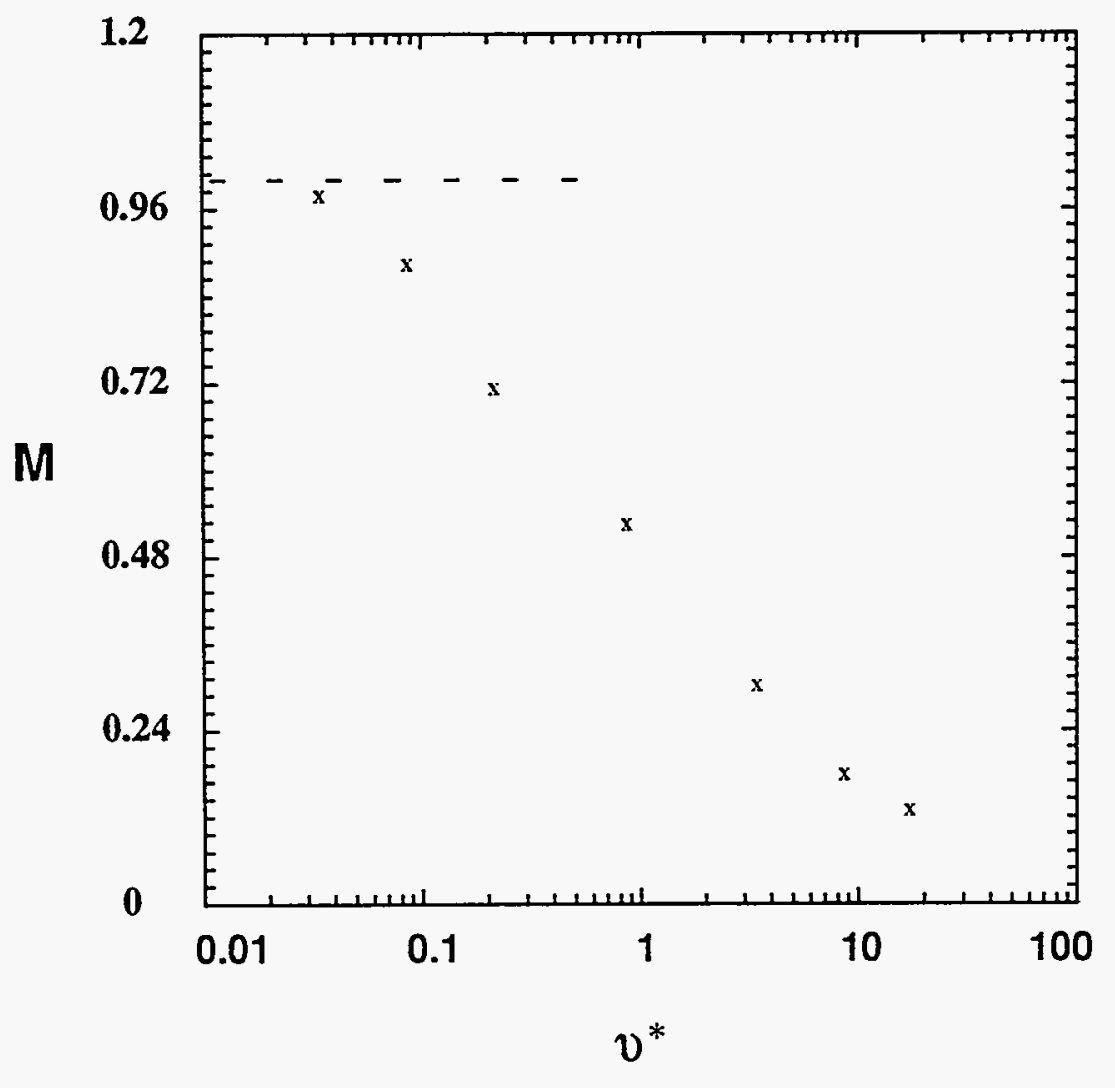

Figure 16: 


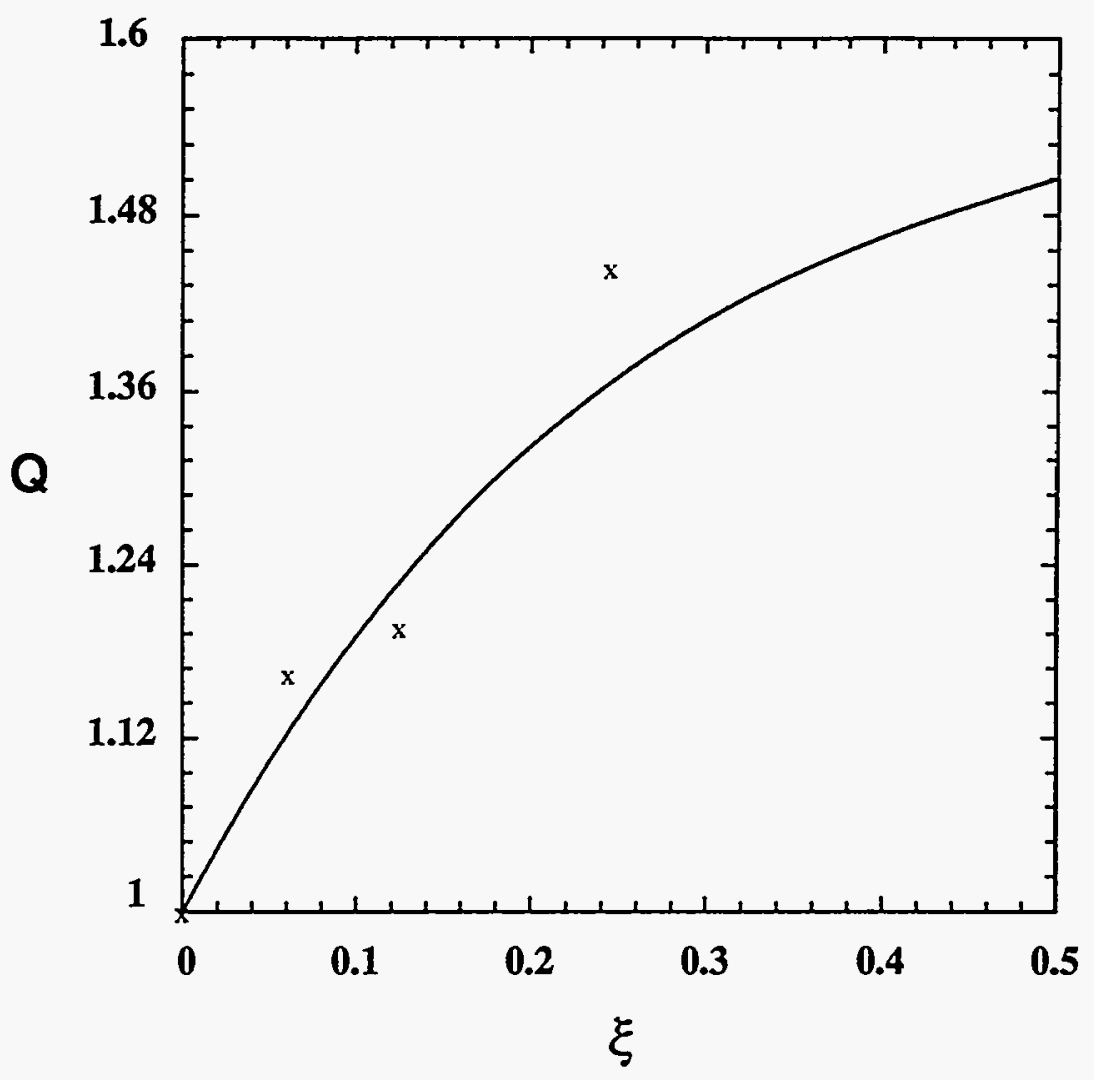

Figure 17: 


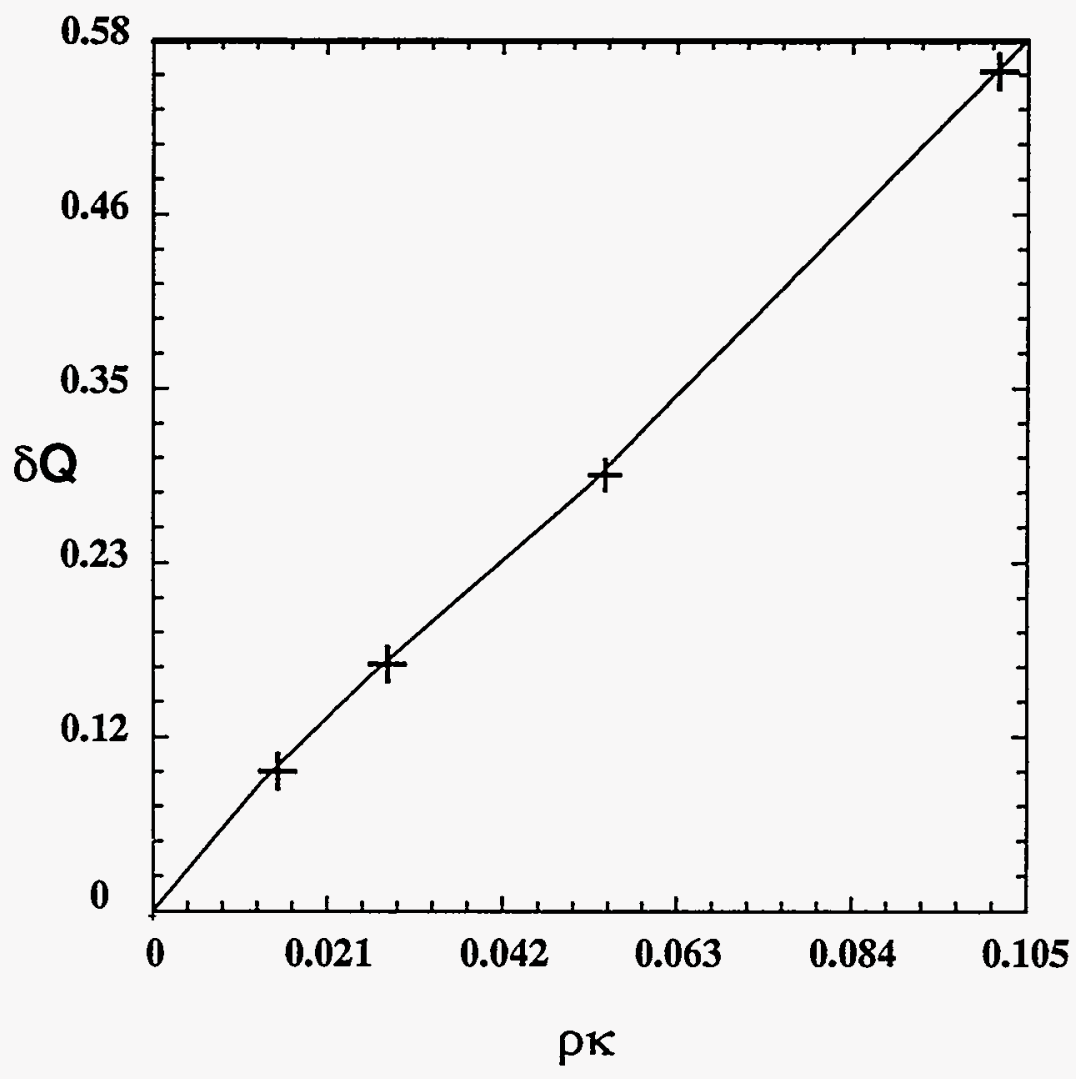

Figure 18: 


\section{EXTERNAL DISTRIBUTION IN ADDITION TO UC-420}

Dr. F. Paoloni, Univ. of Wollongong, AUSTRALIA

Prot. R.C. Cross, Univ. of Sydney, AUSTRALIA

Plasma Research Lab., Australian Nat. Univ., AUSTRALIA

Prof. 1.R. Jones, Flinders Univ, AUSTAALIA

Prof. F. Cap, Inst. for Theoretical Physics, AUSTRIA

Prof. M. Heindler, Institut fur Theoretische Physik, AUSTRIA

Prof. M. Goossens, Astronomisch instituut, BELGIUM

Ecole Royale Militaire, Lab. de Phy. Plasmas, BELGIUM

Commission-European, DG. XII-Fusion Prog., BELGIUM

Prof. R. Bouciqué, Rijksuniversiteit Gent, BELGIUM

Dr. P.H. Sakanaka, Instituto Fisica, BRAZIL

Prof. Dr. I.C. Nascimento, Instituto Fisica, Sao Paulo, BRAZIL Instituto Nacional De Pesquisas Espaciais-INPE, BRAZIL

Documents Office, Atomic Energy of Canada Ltd., CANADA

Ms. M. Morin, CCFMTTokamak de Varennes, CANADA

Dr. M.P. Bachynski, MPB Technologies, Inc., CANADA

Dr. H.M. Skarsgard, Univ. of Saskatchewan, CANADA

Prof. J. Teichmann, Univ. of Montreal, CANADA

Prof. S.R. Sreenivasan, Univ. of Calgary, CANADA

Prof. R. Marchand, INRS-Energie et Materiaux, CANADA

Dr. R. Bolton, Centre canadien de fusion magnétique, CANADA

Dr. C.R. James., Univ. of Alberta, CANADA

Dr. P. Lukác, Komenského Universzita, CZECHO-SLOVAKIA

The Librarian, Culham Laboratory, ENGLAND

Library, R61, Ruthertord Appleton Laboratory, ENGLAND

Mrs. S.A. Hutchinson, JET Library, ENGLAND

Dr. S.C. Shama, Univ. of South Pacific, FIJI ISLANDS

P. Măhönen, Univ. of Helsinki, FINLAND

Prof. M.N. Bussac, Ecole Polytechnique., FRANCE

C. Mouttet, Lab. de Physique des Milieux lonisés, FRANCE

J. Radet, CEN/CADARACHE - Bat 506, FRANCE

Prof. E. Economou, Univ. of Crete, GREECE

Ms. C. Rinni, Univ. of loannina, GREECE

Preprint Library, Hungarian Academy of Sci., HUNGARY

Dr. B. DasGupta, Saha Inst. of Nuclear Physics, INDIA

Dr. P. Kaw, Inst. for Plasma Research, INDIA

Dr. P. Rosenau, Israel Inst. of Technology, ISRAEL

Librarian, Intemational Center for Theo Physics, ITALY

Miss C. De Palo, Associazione EURATOM-ENEA , ITALY

Dr. G. Grosso, Istituto di Fisica del Plasma, ITALY

Prof. G. Rostangni, Istituto Gas lonizzati Del Cnr, ITALY
Dr. H. Yamato, Toshiba Res \& Devel Center, JAPAN

Prof. I. Kawakami, Hiroshima Univ., JAPAN

Prof. K. Nishikawa, Hiroshima Univ., JAPAN

Librarian, Naka Fusion Research Establishment, JAERI, JAPAN

Director, Japan Atomic Energy Research Inst., JAPAN

Prof. S. Itoh, Kyushu Univ., JAPAN

Research Info. Ctr., National Instit. for Fusion Science, JAPAN

Prof. S. Tanaka, Kyoto Univ., JAPAN

Library, Kyoto Univ., JAPAN

Prof. N. Inous, Univ. of Tokyo, JAPAN

Secrotary, Plasma Section, Electrotechnical Lab., JAPAN

Dr. O. Mitarai, Kumamoto Inst. of Technology, JAPAN

Dr. G.S. Lee, Korea Basic Sci. Ctr., KOREA

J. Hyeon-Sook, Korea Atomic Energy Research Inst., KOREA

D.I. Choi, The Korea Adv. Inst. of Sci. \& Tech., KOREA

Prof. B.S. Liley, Univ. of Waikato, NEW ZEALAND

Inst of Physics, Chinese Acad Sci PEOPLE'S REP. OF CHINA

Library, Inst. of Plasma Physics, PEOPLE'S REP. OF CHINA

Tsinghua Univ. Library, PEOPLE'S REPUBLIC OF CHINA

Z. Li, S.W. Inst Physics, PEOPLE'S REPUBLIC OF CHINA

Prof. J.A.C. Cabral, Instituto Superior Tecnico, PORTUGAL

Prof. M.A. Hellberg, Univ. of Natal, S. AFRICA

Prof. D.E. Kim, Pohang Inst. of Sci. \& Tech., SO. KOREA

Prof. C.I.E.M.A.T. Fusion Division Library, SPAIN

Dr. L. Stenflo, Univ. of UMEA, SWEDEN

Library, Royal Inst. of Technology, SWEDEN

Prof. H. Wilhelmson, Chalmers Univ. of Tech., SWEDEN

Centre Phys. Des Plasmas, Ecole Polytech, SWITZERLAND

Bibliotheek, Inst. Voor Plasma-Fysica, THE NETHERLANDS

Asst. Prof. Dr. S. Cakir, Middle East Tech. Univ., TURKEY

Dr. V.A. Glukhikh,Sci. Res. Inst. Electrophys.l Apparatus, USSR

Dr. D.D. Ryutov, Siberian Branch of Academy of Sci., USSR

Dr. G.A. Eliseev, I.V. Kurchatov Inst., USSR

Librarian, The Ukr.SSR Academy of Sciences, USSR

Dr. L.M. Kovrizhnykh, Inst. of General Physics, USSR

Kemforschungsanlage GmbH, Zentralbibliothek, W. GERMANY

Bibliothek, Inst. Fur Plasmaforschung, W. GERMANY

Prof. K. Schindler, Ruhr-Universitát Bochum, W. GERMANY

Dr. F. Wagner, (ASDEX), Max-Planck-Institut, W. GERMANY

Librarian, Max-Planck-Institut, W. GERMANY 